\title{
Neutral carbon and $\mathrm{CO}$ emission in the core and the halo of dark cloud Barnard 5
}

\author{
F. Bensch ${ }^{1,2}$ \\ 1 Radioastronomisches Institut, Universität Bonn (RAIUB), Auf dem Hügel 71, 53121 Bonn, Germany \\ e-mail: fbensch@astro.uni-bonn.de \\ ${ }^{2}$ Harvard-Smithsonian Center for Astrophysics (CfA), 60 Garden Street, Cambridge, MA 02138, USA
}

Received 6 June 2005 / Accepted 11 October 2005

ABSTRACT

\begin{abstract}
Aims. The physical conditions and chemical structure in the dark cloud of Barnard 5 and its surrounding atomic halo is studied. The impact of the halo on the line emission emerging from the molecular cloud is investigated.

Methods. We present observations of the $[\mathrm{CI}]{ }^{3} \mathrm{P}_{1} \rightarrow{ }^{3} \mathrm{P}_{0}$ transition of neutral carbon and the low- $J$ transitions of ${ }^{12} \mathrm{CO}$ and ${ }^{13} \mathrm{CO}$. The $\mathrm{CO}$ maps extend from the core $\left(A_{\mathrm{v}} \gtrsim 7\right)$ to the northern cloud edge and into the halo $\left(A_{\mathrm{v}} \lesssim 1\right)$. They are complemented by deeply integrated [CI] spectra made along a 1D cut of similar extent. Escape probability and photon-dominated region (PDR) models are employed to interpret the observations.

Results. ${ }^{12} \mathrm{CO}$ and ${ }^{13} \mathrm{CO}$ are detected in the cloud and the halo, while $[\mathrm{CI}]$ is detected only toward the molecular cloud. This occurs even though the neutral carbon column density is $\gtrsim 5$ times larger than the $\mathrm{CO}$ column density in the halo, but it can be understood in terms of excitation. The $[\mathrm{CI}]$ excitation is governed by collisions even at the low halo densities, while the $\mathrm{CO}$ excitation is dominated by the absorption of line photons emitted by the nearby molecular cloud. The upper limit on the neutral carbon column density in the halo is $6 \times 10^{15} \mathrm{~cm}^{-2}$.

The PDR studies show that even small column densities of $\mathrm{H}_{2}$ and $\mathrm{CO}$, such as those in the $\mathrm{B} 5$ halo, can significantly change the [CI] and $\mathrm{CO}$ line emission (pre-shielding). Since this effect decreases the $[\mathrm{CI}]$ intensity and increases the $\mathrm{CO}$ intensity, the largest impact is noted for the $[\mathrm{CI}] / \mathrm{CO}$ line ratios. For the B5 cloud, a PDR model with a molecular hydrogen column density of $\sim 6 \times 10^{19} \mathrm{~cm}^{-2}$ in the halo matches the observed $[\mathrm{CI}] / \mathrm{CO}$ line ratios best. Models with no pre-shielding, in contrast, suggest high gas densities that are in conflict with independently derived densities. The PDR models with a $\chi<1$ demonstrate that the $[\mathrm{CI}] / \mathrm{CO}$ ratios cannot be attributed solely to a reduced FUV field.
\end{abstract}

Key words. ISM: abundances - astrochemistry - ISM: clouds - ISM: individual: B5 - radio lines: ISM

\section{Introduction}

Dark clouds are noted at optical wavelengths by their absorption of the stellar background, showing that they have a column density of at least several magnitudes in visible extinction $\left(A_{\mathrm{v}}\right)$. Many of the dark clouds are relatively quiescent with either no active star formation or low-mass star formation proceeding in a few isolated cores. Gas heating is provided by the diffuse interstellar radiation field (ISRF) via photo-electric heating and by cosmic rays for the FUV-shielded parts of the cloud. Protostars embedded in dense cores can heat the gas locally, and the interaction of a linear jet emerging from the protostar with the parental cloud can give rise to shock-heated molecular gas and drive a molecular outflow. However, these processes have generally not had a significant impact on the global thermal balance of the gas, while the cloud is recognized as a dark cloud.

Far ultraviolet photons of the ISRF dominate the chemistry at the dark cloud surface, dissociating and ionizing the molecular gas. This results in a $\mathrm{C}^{+} / \mathrm{C}^{0} / \mathrm{CO}$ transition layer at optical depths below a few magnitudes, while the submillimeter transitions of these species are responsible for much of the gas cooling. Frequent gas-grain collisions can provide additional cooling for high density gas in shielded regions.

Molecular clouds are embedded in the diffuse interstellar medium (ISM) of the Galaxy where most of the gas is in an atomic form. The atomic gas is mostly located in two phases that are in pressure equilibrium, the cold neutral medium (CNM) with temperatures at $T \sim 100 \mathrm{~K}$ and the warm neutral medium (WNM) with $T \sim 10^{4} \mathrm{~K}$ (for example, Wolfire et al. 2003, and the reviews by Kulkarni \& Heiles 1987; Dickey \& Lockman 1990; McKee et al. 2004). Some of the cold, atomic gas is associated with the denser molecular clouds, possibly forming an extended halo or envelope (Andersson et al. 1992; Andersson \& Wannier 1993; Moriarty-Schieven et al. 1997). The gas surrounding molecular clouds is rather difficult to probe observationally because of the small column densities and thus the weak emission of the gas. Such halos are expected to have a significantly lower visual extinction (typically, below $1 \mathrm{mag}$ ) and a density of a few ten $\mathrm{cm}^{-3}$, which is smaller 
than measured for molecular clouds. The halo temperature is between a few tens to $100 \mathrm{~K}$, which is warmer than the molecular cloud but colder than the diffuse ISM. Even though we refer to this component as a halo, observational studies suggest that the atomic envelope of molecular clouds is highly structured and filamentary (see, for example, the HI mapping data by Wannier et al. 1999; Moriarty-Schieven et al. 1997) and that their shape and extent can vary considerably for different molecular clouds.

In addition to neutral hydrogen (Dickey \& Lockman 1990) and $\mathrm{H}_{2}$ absorption-line studies at UV wavelengths (Spitzer \& Jenkins 1975; Shull \& Beckwith 1982; Rachford et al. 2002), neutral carbon and $\mathrm{CO}$ are important trace species for the study of molecular clouds and the diffuse gas in the CNM. Low- $J$ $\mathrm{CO}$ transitions are widely used tracers of molecular gas, and even the relatively weak emission of the neutral carbon finestructure transition has been studied extensively for dark clouds (Frerking et al. 1989; Schilke et al. 1995; Stark et al. 1996; Tatematsu et al. 1999; Maezawa et al. 1999; Kamegai et al. 2003). Absorption-line studies of these species at UV wavelengths probe the physical conditions and their abundances in the diffuse neutral gas of the halo: for example, the surveys presented by Federman et al. (1980), Jenkins et al. (1983), Jenkins \& Tripp (2001), in addition to numerous studies made for individual lines-of-sight. These UV absorption-line studies are currently not possible for gas with high visual extinction, such as dark clouds. The sensitivity of most sub-mm [CI] observations, in contrast, are limited to column densities that are larger than a few times $10^{16} \mathrm{~cm}^{-2}$, unless very deeply integrated observations are done, which are difficult to obtain with ground-based telescopes. The detection of the sub-mm emission of neutral carbon in neutral gas halo is an important goal, however, because it cross-calibrates the results obtained from UV absorption measurements (Keene et al. 1987).

Despite the low column densities and weak emission of the CNM in the halo, the halo gas potentially has a significant impact on the chemistry in molecular cloud surfaces and thus on the line emission of neutral carbon and $\mathrm{CO}$ emerging from molecular clouds. The presence of even a small column density of molecular hydrogen and $\mathrm{CO}$ in the halo, such as detected for B5 (Wannier et al. 1999), can provide a significant shielding for the CO in the molecular cloud (van Dishoeck \& Black 1988). In Bensch et al. (2003) we suggested that this effect might be responsible for the surprisingly low $[\mathrm{CI}] / \mathrm{CO}$ ratios observed in MCLD $123.5+24.9$ and other translucent clouds with only a little dust shielding against the ISRF. For a test of this hypothesis, the $\mathrm{CO}$ and $[\mathrm{CI}]$ emission in Barnard 5 is considered as a typical example of a dark cloud. The spectral line emission is observed for a region that stretches from the cloud core into the surrounding halo. In addition, we have searched for sub$\mathrm{mm}[\mathrm{CI}]$ emission in the diffuse halo. This allows for a comparison of the neutral carbon column densities derived from the radio observations to those from UV absorption-line data. Section 2 summarizes the literature on B5 that is relevant for the present study. The spectral-line observations are presented in Sect. 3 and the most salient features of the observed emission discussed in Sect. 4. The excitation of $\mathrm{CO}$ and neutral carbon in the molecular cloud and the surrounding halo is discussed in
Sect. 5. Photon-Dominated Region (PDR) model simulations are presented in Sect. 6, and Sect. 7 summarizes the results.

\section{Molecular cloud B5}

Barnard 5 (=Lynds dark cloud 1471) is a relatively small and isolated cloud in the easternmost part of the Perseus molecular cloud complex. Distance estimates for B5 range between 160 pc (Young et al. 1982) and $\geq 350$ pc (Herbig \& Jones 1983; Dickman 1978). For the remainder of this paper $d=260 \mathrm{pc}$ is adopted, which is the distance derived by Cernis (1993) using the extinction determined from photometric observations made for stars toward the eastern part of the Perseus cloud complex.

Several authors (Langer et al. 1989; Yu et al. 1999; Goodman 2004; Sun et al. 2005) have reported extensive mapping observations of the rotational-line emission of $\mathrm{CO}$ and its isotopologues ${ }^{13} \mathrm{CO}, \mathrm{C}^{18} \mathrm{O}$. These maps have an angular resolution between 0.5 and 2.0 and show a molecular cloud shaped like a trapezoid. The angular extent of the cloud ${ }^{1}$ is $\sim 1^{\circ} \times 0.5^{\circ}$, corresponding to a linear size of $\sim 4.4 \times 2.3 \mathrm{pc}$ at a distance of $260 \mathrm{pc}$. Langer et al. (1989) estimate a total cloud mass of $930 M_{\odot}$ and a virial mass of $1390 M_{\odot}$ using ${ }^{13} \mathrm{CO}$ $J=1 \rightarrow 0$ observations and assuming $d=350$ pc. This corresponds to $M_{\text {cloud }} \approx 510 M_{\odot}$ and $M_{\text {vir }} \approx 1000 M_{\odot}$ for the distance of $d=260 \mathrm{pc}$ adopted in the present paper. Young et al. (1982) derive a kinetic temperature between $10-15 \mathrm{~K}$ and an average density of $n \sim 1.7 \times 10^{3} \mathrm{~cm}^{-3}$ for the shielded molecular gas using multi-line $\mathrm{CO}$ observations. Similar temperatures of $T=12-14 \mathrm{~K}$ are noted by Langer et al. (1989). The $\mathrm{C}^{18} \mathrm{O}$ observations by Goldsmith, Langer \& Wilson (1986) probe the $\mathrm{CO}$ gas deeper in the cloud and reveal a clumpy density structure with gas densities between $(3-7) \times 10^{3} \mathrm{~cm}^{-3}$ for the clumps. The visual extinction reaches $A_{\mathrm{v}} \gtrsim 7 \mathrm{mag}$ toward the cloud center (Fig. 1). Observations of transitions with a high critical density for collisional excitation, such as CS, $\mathrm{HCN}$, and $\mathrm{NH}_{3}$, show a core with density close to or exceeding $10^{4} \mathrm{~cm}^{-3}$ in the region with the highest extinction (Benson \& Myers 1989; Zhou et al. 1989; Fuller et al. 1991).

Four point sources were detected with the IRAS satellite, indicating that B5 is actively forming low-mass stars (Beichman et al. 1984). The most luminous of the IRAS point sources, IRS 1, is located in the dense core and drives a highly collimated CO outflow. The outflow is oriented approximately in a NE-SW direction and has a projected length of $\sim 0.5^{\circ}$, a significant fraction of the cloud's angular extent (Fuller et al. 1991; Yu et al. 1999). However, the total luminosity of the embedded IRAS sources is low, and these objects do not significantly heat the gas in the cloud. In fact, the IRAS dust emission maps presented by Langer et al. (1989) show that B5 is externally heated by the diffuse ISRF. This is indicated by the higher dust temperature at the cloud surface, derived from the IRAS $60 \mu \mathrm{m}$ and $100 \mu \mathrm{m}$ bands and the limb-brightened $12 \mu \mathrm{m}$ emission. Similarly, analysis of the CO line excitation along cuts across the cloud edge by Young et al. (1982) suggests a

\footnotetext{
${ }^{1}$ Defined, for example, by the $10 \%$ contour of the peak ${ }^{12} \mathrm{CO} J=1 \rightarrow 0$ line-integrated intensity.
} 

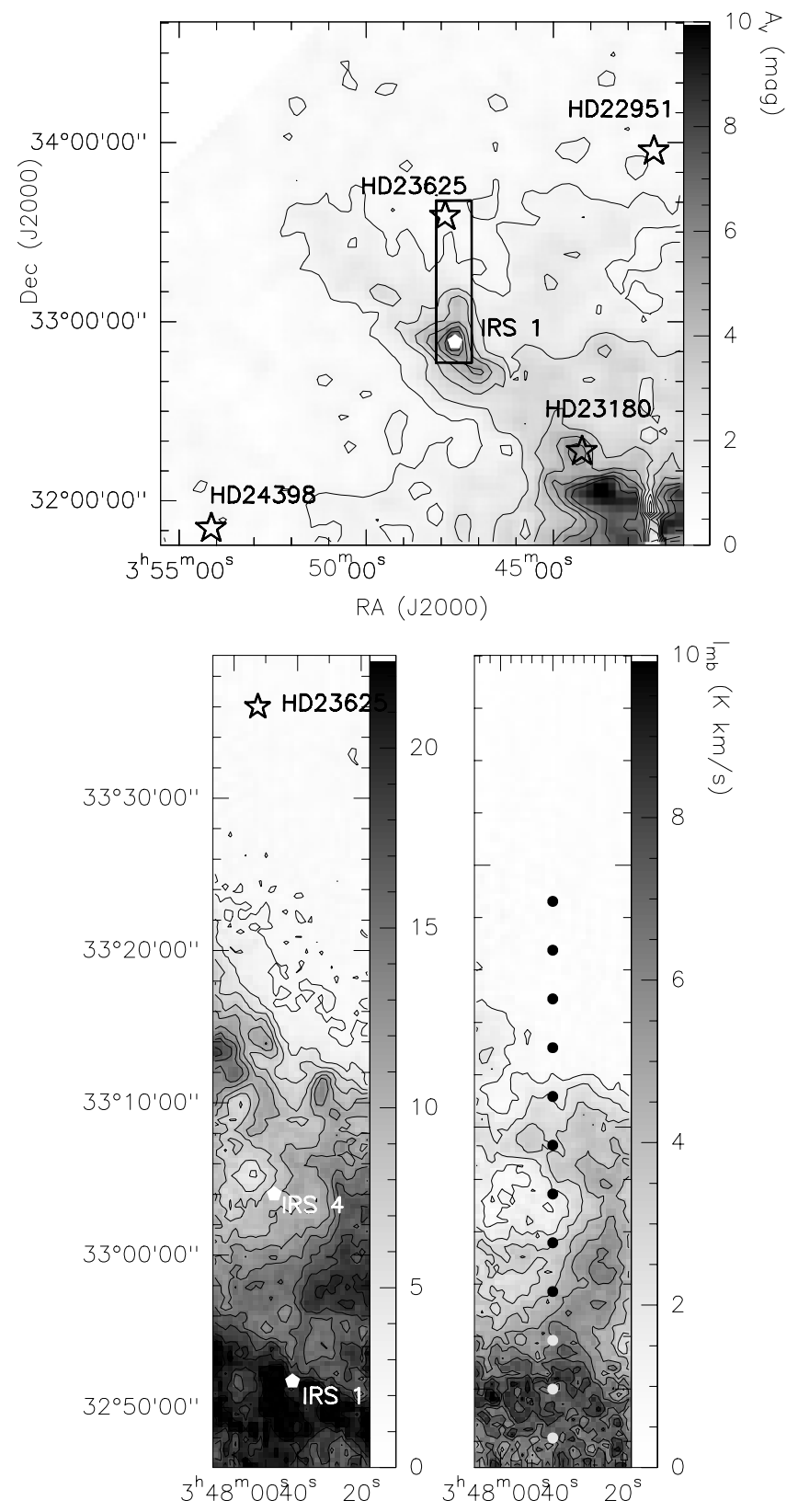

Fig. 1. Top panel: 2MASS-based "NICER" extinction map toward B5 (COMPLETE Project 2004). The extinction is given in visual magnitudes $\left(A_{\mathrm{v}}\right)$, and contours are drawn from 1 to $7 \mathrm{mag}$, in steps of $1 \mathrm{mag}$. The background stars toward which absorption-line observations are presented by Savage et al. (1977) and Wannier et al. (1999) are indicated by their Henry Draper Catalogue number. Bottom left panel: ${ }^{12} \mathrm{CO} J=1 \rightarrow 0$ line-integrated intensity, $I_{\mathrm{mb}, \mathrm{c}}=\int T_{\mathrm{mb}, \mathrm{c}} \mathrm{d} v$, in $\mathrm{K} \mathrm{km} \mathrm{s}^{-1}$. Contours are drawn from 2 to $20 \mathrm{~K} \mathrm{~km} \mathrm{~s}^{-1}$, in steps of $2 \mathrm{~K} \mathrm{~km} \mathrm{~s}^{-1}$. The position of the IRAS point sources B5 IRS 1 and IRS 4 are marked by white pentagons. Bottom right panel: ${ }^{13} \mathrm{CO} J=1 \rightarrow 0$ line-integrated intensity (contours from 1 to $10 \mathrm{~K} \mathrm{~km} \mathrm{~s}^{-1}$, in steps of $1 \mathrm{~K} \mathrm{~km} \mathrm{~s}^{-1}$ ). Dots indicate the pointings of the SWAS $[\mathrm{CI}]{ }^{3} \mathrm{P}_{1} \rightarrow{ }^{3} \mathrm{P}_{0}$ observations.

higher excitation temperature for $\mathrm{CO}$ at the cloud surface with $T \gtrsim 25 \mathrm{~K}$, indicative of externally heated gas.

The low- $A_{\mathrm{v}}$ gas surrounding the B5 dark cloud was studied at radio frequencies using observations of the $\mathrm{HI}$ emission, and at visible and UV wavelengths by absorption-line studies toward background stars. Andersson et al. (1992) present observations of the $21 \mathrm{~cm} \mathrm{HI}$ emission made with the DRAO interferometer and estimate the total mass in the diffuse halo to be $350 M_{\odot}$. The visual extinction measured toward the halo is typically between 0.5-1 mag (Černis 1993, COMPLETE Project 2004). Absorption-line studies at visible and UV wavelengths toward background stars with a projected distance of $\lesssim 2^{\circ}$ to the cloud (top panel of Fig. 1) yield both column densities and the excitation condition of neutral and molecular hydrogen (Savage et al. 1977), $C^{0}$, and CO (Wannier et al. 1999), among other species. Typical column densities are $N_{\mathrm{H}}=(6.5-11) \times 10^{20}$, $N_{\mathrm{H}_{2}}=(2.9-4.7) \times 10^{20}, N_{\mathrm{C}^{0}}=(17-48) \times 10^{14}$, and $N_{\mathrm{CO}}=$ $(1.7-8.4) \times 10^{14}$, respectively. This suggests that the halo predominantly consists of atomic gas, but also contains a significant amount of molecular hydrogen with $N_{\mathrm{H}_{2}} \lesssim N_{\mathrm{H}}$. As a low- $A_{\mathrm{v}}$ region exposed to the interstellar radiation field, the dominant carbon species in the halo is expected to be $\mathrm{C}^{+}$, with $N_{\mathrm{C}^{+}} \gg N_{\mathrm{C}} \gg N_{\mathrm{CO}}$. The neutral gas density and temperature derived from the $\mathrm{HI}$ emission and absorption-line studies give $n=35-100 \mathrm{~cm}^{-3}$ and $T_{\text {kin }}=20-60 \mathrm{~K}$, respectively (Savage et al. 1977; Andersson et al. 1992; Wannier et al. 1999). The authors of the last two publications suggest that the neutral halo results from a general outflow of gas due to the higher pressure of the molecular cloud.

\section{Spectral line observations}

Spectral line observations were made for the three lowest rotational transitions of ${ }^{12} \mathrm{CO}$ and ${ }^{13} \mathrm{CO}$ and the $[\mathrm{CI}]{ }^{3} \mathrm{P}_{1} \rightarrow{ }^{3} \mathrm{P}_{0}$ fine-structure transition of neutral carbon, hereafter simply referred to as [CI], unless noted otherwise. The observations cover strips that are elongated in declination and that extend from the cloud core near IRS 1 across the cloud edge and into the surrounding halo (Fig. 1 and Table 1).

\subsection{Ground-state CO transitions}

The ${ }^{12} \mathrm{CO}$ and ${ }^{13} \mathrm{CO} J=1 \rightarrow 0$ ground-state transitions were mapped with the FCRAO $14 \mathrm{~m}$ telescope using the 32 pixel SEQUOIA receiver array (OTF mapping mode). A linear baseline is fitted and subtracted from the spectra and the observations are re-gridded on a $20^{\prime \prime}$ grid. The final maps cover an area of $10.3 \times 53 ! 3$, centered on $\alpha=3^{\mathrm{h}} 47^{\mathrm{m}} 42^{\mathrm{s}}$, and extend from $\delta=+32^{\circ} 46^{\prime} 13^{\prime \prime}$ to $\delta=+33^{\circ} 39^{\prime} 13^{\prime \prime}$ (all co-ordinates given for epoch J2000.0). Thus, the maps cover an area on the sky from IRS 1 in the B5 cloud core to HD 23625, the latter being one of the stars in the absorption-line study by Wannier et al. (1999). Emission is detected for both isotopologues across the entire map, even though the faint emission at $\delta \gtrsim 33^{\circ} 20^{\prime}$ becomes obvious only after the spectra have been smoothed to a lower angular resolution (see Sect. 4 below).

The main problem encountered during the FCRAO observations was to find a nearby reference position that shows no significant emission at a level of $\Delta T_{\mathrm{A}}^{*} \sim 30 \mathrm{mK}$, the rms noise of the FCRAO spectra smoothed to the $\sim 4.3$ angular 
Table 1. Spectral line observations made toward B5.

\begin{tabular}{cccccccc}
\hline \hline transition & telescope & $\begin{array}{c}\theta_{\mathrm{mb}} \\
{[\operatorname{arcsec}]}\end{array}$ & $\begin{array}{c}B_{\text {eff }} / F_{\text {eff }} \\
\approx \eta_{\mathrm{mb}}^{b}\end{array}$ & $\begin{array}{c}\text { map } \\
{\left[\operatorname{arcmin}^{2}\right]}\end{array}$ & $\begin{array}{c}\text { sampling } \\
{[\operatorname{arcmin}]}\end{array}$ & $\begin{array}{c}\text { \# pointings } \\
\text { in map }\end{array}$ & $\begin{array}{c}\Delta v^{a} \\
{\left[\mathrm{~km} \mathrm{~s}^{-1}\right]}\end{array}$ \\
\hline${ }^{12} \mathrm{CO} J=1 \rightarrow 0$ & FCRAO & 44 & 0.45 & $10.3 \times 53.3$ & 0.33 & $31 \times 160$ & 0.1 \\
${ }^{12} \mathrm{CO} J=2 \rightarrow 1$ & KOSMA & 130 & 0.76 & $7.0 \times 38.0$ & 1.0 & $7 \times 38$ & 0.1 \\
${ }^{12} \mathrm{CO} J=3 \rightarrow 2$ & KOSMA & 82 & 0.78 & $7.0 \times 38.0$ & 1.0 & $7 \times 38$ & 0.35 \\
${ }^{13} \mathrm{CO} J=1 \rightarrow 0$ & FCRAO & 44 & 0.50 & $10.3 \times 53.3$ & 0.33 & $31 \times 160$ & 0.1 \\
${ }^{13} \mathrm{CO} J=2 \rightarrow 1$ & KOSMA & 130 & 0.76 & $7.0 \times 38.0$ & 1.0 & $7 \times 38$ & 0.1 \\
${ }^{13} \mathrm{CO} J=3 \rightarrow 2$ & KOSMA & 82 & 0.78 & $7.0 \times 38.0$ & 1.0 & $7 \times 38$ & 0.35 \\
${ }^{13} \mathrm{CO} J=5 \rightarrow 4$ & SWAS & $200 \times 270$ & 0.90 & $-{ }^{c}$ & 3.2 & $1 \times 12$ & 0.62 \\
${ }^{[\mathrm{CI}]}{ }^{3} \mathrm{P}_{1} \rightarrow{ }^{3} \mathrm{P}_{0}$ & SWAS & $210 \times 300$ & 0.90 & $-{ }^{c}$ & 3.2 & $1 \times 12$ & 0.62 \\
\hline
\end{tabular}

${ }^{a}$ Velocity channel width.

${ }^{b}$ Note that the FCRAO and SWAS give the beam efficiency as $\eta_{\mathrm{mb}}$, while $F_{\text {eff }}, B_{\text {eff }}$ are quoted for the KOSMA telescope. In this context $\eta_{\mathrm{mb}}$ is equivalent to $\left(B_{\text {eff }} / F_{\text {eff }}\right)$.

${ }^{c}$ Single strip of 12 pointings.

resolution of the SWAS beam. The reference position ${ }^{2}$ near $\alpha=3^{\mathrm{h}} 51^{\mathrm{m}} 39^{\mathrm{s}} \delta=31^{\circ} 28^{\prime} 58^{\prime \prime}$, which was used for the initial observing run, shows emission with $T_{\mathrm{A}}^{*} \sim 0.5 \mathrm{~K}$ and a line-integrated intensity of $\sim 0.4 \mathrm{~K} \mathrm{~km} \mathrm{~s}^{-1}$. The northern half of the map was therefore re-observed using a second reference position near $\alpha=3^{\mathrm{h}} 56^{\mathrm{m}} 29^{\mathrm{s}}, \delta=35^{\circ} 04^{\prime} 51^{\prime \prime}$, the position of HD 24640. This is the line-of-sight with the smallest visual extinction in the list compiled by Kaczmarczyk (2000), and the region is free of $\mathrm{CO}$ emission down to a level of $T_{\mathrm{A}}^{*}<73 \mathrm{mK}(3 \sigma)$.

The main beam efficiency of the FCRAO telescope is $\eta_{\mathrm{mb}}=$ 0.45 at the line frequency of the ${ }^{12} \mathrm{CO} J=1 \rightarrow 0$ transition and 0.5 for ${ }^{13} \mathrm{CO} J=1 \rightarrow 0$ transition, respectively, where $\eta_{\mathrm{mb}}$ was taken from the FCRAO web-site ${ }^{3}$ and is equivalent to the ratio $B_{\text {eff }} / F_{\text {eff }}$ used for the KOSMA observations. The relatively low $\eta_{\mathrm{mb}}$ indicates that an extended stray- and error-beam pattern might play a role for the detected emission. In particular, a significant error beam pick-up is expected to apply to the ${ }^{12} \mathrm{CO}$ observations because of the relatively strong and extended emission of the main isotopologue. While the $T_{\mathrm{mb}}$ scale probably overestimates the intensity detected by the main beam, the antenna temperature scale underestimates it, because only a part of the error-beam pattern is terminated on the line-emitting cloud. The correction for the error beam pick-up requires knowledge of the size and amplitude of the beam pattern, which is not available for the FCRAO telescope. The pragmatic solution employed here is to calculate the average of the spectrum scaled to the antenna temperature and the main beam temperature, $T_{\mathrm{mb}, \mathrm{c}} \approx 0.5\left(T_{\mathrm{mb}}+T_{\mathrm{A}}^{*}\right)$. In this context, the error beam-corrected temperature scale is denoted as $T_{\mathrm{mb}, \mathrm{c}}$ (Bensch et al. 2001a). The accuracy of the spectra scaled in this fashion is then given by half of the difference between both intensity scales, $\Delta T_{\mathrm{mb}, \mathrm{c}} \approx 0.5\left(T_{\mathrm{mb}}-T_{\mathrm{A}}^{*}\right)$. This gives us $T_{\mathrm{mb}, \mathrm{c}} \approx T_{\mathrm{A}}^{*} / 0.62$ and $\Delta T_{\mathrm{mb}, \mathrm{c}} / T_{\mathrm{mb}, \mathrm{c}} \approx 38 \%$ for the FCRAO observations at $115 \mathrm{GHz}$, and $T_{\mathrm{mb}, \mathrm{c}} \approx T_{\mathrm{A}}^{*} / 0.67$ with a slightly

\footnotetext{
${ }^{2}$ Note that the SEQUOIA array covers an area of $5^{\prime} \times 5^{\prime}$ on the sky and is fixed in Azimuth-Elevation. In addition, several OTF scans made at different elevations were re-gridded and co-added for the final map. Therefore, a single, well-defined off-source position cannot be assigned to the spectra in the map.

${ }^{3}$ http://www. astro. umass. edu/ fcrao/observer
}

smaller systematic error of $\Delta T_{\mathrm{mb}, \mathrm{c}} / T_{\mathrm{mb}, \mathrm{c}} \approx 34 \%$ for $110 \mathrm{GHz}$. Note, however, that an error-beam pick also modifies the line profile, which is not corrected with this treatment (see Bensch et al. 2001b for a more detailed discussion).

\subsection{KOSMA CO $J=2 \rightarrow 1$ and $J=3 \rightarrow 2$ observations}

The mapping observations for the higher ${ }^{12} \mathrm{CO}$ and ${ }^{13} \mathrm{CO}$ rotational transitions were made with the KOSMA $3 \mathrm{~m}$ telescope, located on Gornergrat, Switzerland. The KOSMA observations have a lower angular resolution than the FCRAO data and were made with a 1 arcmin sampling (Table 1). The on-source integration time per position varied between $\sim 1$ min toward the cloud core and $2.5 \mathrm{~min}$ for positions in the northern part of the map where the CO emission is much weaker. The KOSMA maps are centered on the FCRAO map in Right Ascension $\left(\alpha=3^{\mathrm{h}} 47^{\mathrm{m}} 42^{\mathrm{s}}\right)$ but have a smaller extent in declination, from $\delta=32^{\circ} 48^{\prime} 13^{\prime \prime}$ to $\delta=33^{\circ} 25^{\prime} 13^{\prime \prime}$. The observations for the $J=2 \rightarrow 1$ and $J=3 \rightarrow 2$ transitions were made simultaneously using the Dual-channel-SIS receiver. The telescope pointing was checked frequently with an optical pointing telescope. The alignment of the optical pointing with the radio beam was checked using continuum observations on planets made with the low-frequency $(230 \mathrm{GHz})$ channel of the radio receiver. Because of a small misalignment of both receiver channels, the pointing of the $345 \mathrm{GHz}$ beam is offset by $\triangle \mathrm{AZI}=+7^{\prime \prime}, \triangle \mathrm{ELE}=-28^{\prime \prime}$ on the sky relative to the low-frequency beam. This $\sim 0.5$ offset was not corrected in the resulting maps because only those $\mathrm{CO}$ observations that are smoothed to the much larger angular resolution of the SWAS beam $(\sim 4 ! 3)$ are used in the present paper.

The KOSMA observations use the off-source position at $\alpha=3^{\mathrm{h}} 47^{\mathrm{m}} 42^{\mathrm{s}}, \delta=32^{\circ} 51^{\prime} 13^{\prime \prime}$, and no contamination is evident in the spectra to a level of $\Delta T_{\mathrm{A}}^{*} \sim 110 \mathrm{mK}$ for ${ }^{12} \mathrm{CO} J=2 \rightarrow 1$ and $\Delta T_{\mathrm{A}}^{*} \sim 160 \mathrm{mK}$ for ${ }^{12} \mathrm{CO} J=3 \rightarrow 2$, respectively. This was derived as the $3 \sigma$ upper limit from the rms noise per velocity channel of the KOSMA spectra smoothed to the 4.3 angular resolution.

A significant error beam pick-up is not expected for the data because of the relatively large main beam efficiency of the KOSMA telescope at the CO $J=2 \rightarrow 1$ and 
$J=3 \rightarrow 2$ line frequencies. The spectra are therefore scaled to the main beam brightness temperature following $T_{\mathrm{mb}}=$ $T_{\mathrm{A}}^{*}\left(B_{\mathrm{eff}} / F_{\mathrm{eff}}\right)^{-1}$ (Downes 1989$)$ and using the ratio $B_{\text {eff }} / F_{\text {eff }}$ listed in Table 1 . The systematic error of the line intensity is estimated in the same way as for the FCRAO observations, giving $\Delta T_{\mathrm{mb}} / T_{\mathrm{mb}} \approx 12 \%$.

\subsection{SWAS [CI] observations}

Observations of the ${ }^{3} \mathrm{P}_{1} \rightarrow{ }^{3} \mathrm{P}_{0}$ ground-state fine-structure transition of neutral carbon were made with the Submillimeter Wave Astronomy Satellite, SWAS ${ }^{4}$. In total, observations were made for 30 pointings along a N-S and E-W strip, centered on $\alpha=3^{\mathrm{h}} 47^{\mathrm{m}} 41^{\mathrm{s}} 6, \delta=32^{\circ} 51^{\prime} 13^{\prime \prime}$. The latter position is denoted as the " $(0,0)$-position" in the following. It is the SWAS pointing with the smallest angular separation from IRS 1 (at $\Delta \alpha=-6$ ", $\Delta \delta=+30^{\prime \prime}$ w.r.t. to the " $(0,0)$-position").

The spacing of the observations is 3.2 , slightly smaller than the minor axis FWHM of the elliptical SWAS beam. In the remainder of the paper, only the spectra made toward the B5 core and the northern half of the N-S strip are discussed, where complementary $\mathrm{CO}$ observations are available (12 positions). They are indicated by the dark dots in the lower right panel of Fig. 1. Again, the integration time is longer for observations with larger declination because of the decreasing line intensity along the cut from the cloud center to the edge. The on-source integration time for each position increased from $\sim 8 \mathrm{~min}$ to $\sim 1 \mathrm{~h}$ toward the cloud $\left(0^{\prime} \leq \Delta \delta \leq 16^{\prime}\right)$, and up to $\sim 20 \mathrm{~h}$ for positions with $\Delta \delta \geq 19$ '.2. A polynomial up to third order was fitted and subtracted from the baseline, and the resulting spectra were scaled to the main beam brightness temperature. The rms noise of the SWAS spectra ranges from $\Delta T_{\mathrm{mb}} \approx 0.2 \mathrm{~K}$ per velocity channel ${ }^{5}$ for positions where emission is detected to $\Delta T_{\mathrm{mb}} \sim 20 \mathrm{mK}$ per channel for spectra toward the halo.

Note that SWAS simultaneously observes the transitions of four species, $[\mathrm{CI}]$ at $492.2 \mathrm{GHz},{ }^{13} \mathrm{CO} J=5 \rightarrow 4$ at $550.9 \mathrm{GHz}$, $\mathrm{H}_{2} \mathrm{O}$ at $556.9 \mathrm{GHz}$, and the $\mathrm{O}_{2}$ at $487.2 \mathrm{GHz}$. No emission was detected for the last three species, however. The rms noise of the ${ }^{13} \mathrm{CO} J=5 \rightarrow 4$ spectra is between $13 \mathrm{mK}$ and $200 \mathrm{mK}$ per velocity channel. The upper limit to the ${ }^{13} \mathrm{CO} J=5 \rightarrow 4$ line intensity turns out to be not very constraining for the physical cloud parameters and the PDR model, however, and is not discussed in the following.

\section{Line emission}

In the following, the peak and line-integrated intensity are derived, and the line ratios determined. These quantities are used in the sections below to derive the excitation conditions of the gas and provide the constraints for the PDR models presented in Sect. 6. For this purpose, the CO spectra are smoothed to

${ }^{4}$ See Melnick et al. (2000) for details on the SWAS satellite and the primary mission objectives. The SWAS data are available online at http://lambda.gsfc.nasa.gov/product/swas/ and http://irsa.ipac. caltech.edu/applications/SWAS/

5 The channel spacing of the SWAS acousto-optical spectrometer is $0.62 \mathrm{~km} \mathrm{~s}^{-1}$; the noise fluctuation band-width is $\sim 1.4 \mathrm{~km} \mathrm{~s}^{-1}$. an angular resolution of 4.3 at the 12 positions where SWAS observations were made. The error introduced with the assumption of a circular SWAS beam for the comparison of the $\mathrm{CO}$ data to the SWAS observations is on the order of a few percent only and thus much smaller than the systematic error estimated for the (corrected) main beam brightness temperature of the CO data. Only the smoothed CO spectra are discussed because the main goal of this paper is to study the large-scale variation of the emission for different impact parameters (offset $\Delta \delta$ ) on the cloud. Figure 2 shows the [CI] and the smoothed CO spectra for 6 of the 12 positions.

For practical reasons, the 12 positions along the N-S strip are grouped into different regions. The "B5 core region" comprises 3 positions with $\Delta \delta \leq 6 ! 2$. The line-integrated intensity CO maps show a depression near IRS 4 , and the 4 positions near that depression $\left(9{ }^{\prime} \cdot 6 \leq \Delta \delta \leq 16^{\prime} 0\right)$ are referred to as the "IRS 4 region". The "cloud edge" is arbitrarily defined as the position $\Delta \delta=22$ ! 4 , just inside the lowest contour in the ${ }^{12} \mathrm{CO}$ $J=1 \rightarrow 0$ map in Fig. 1, where the line-integrated intensity has dropped to $\sim 10 \%$ of its peak value. This is also the largest offset $\Delta \delta$ where emission is detected for all transitions. Offsets $\Delta \delta \geq 25$ '.6 are lumped together as "halo region" (3 positions). This subdivision is rather arbitrary and based more on the sensitivity of the data than on physical grounds; however, it facilitates the discussion below.

\subsection{Spatial variation of the emission}

The peak intensity determined by visual inspection of the spectra is listed in Table 2 (in units of $\mathrm{K}$ ), and the line-integrated intensities along the N-S cut are shown in Fig. $4\left(I=\int T \mathrm{~d} v\right.$,

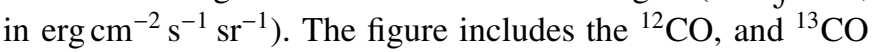
$J=1 \rightarrow 0$ integrated intensity of five additional positions with $\Delta \delta>32^{\prime}$.0. (Recall that the FCRAO map extends in declination up to $+33^{\circ} 39^{\prime}$, and thus farther into the halo than the observations made with KOSMA and SWAS.) These spectra have been smoothed to the same 4.3 angular resolution.

The accuracies of the peak temperature and of the lineintegrated intensities are calculated from the sum of the squared baseline rms noise and the systematic error of the intensity calibration. Even though systematic and statistical errors cannot be combined as independent errors, this procedure gives a reasonable estimate for the overall accuracy of the derived line intensities ${ }^{6}$.

The line-integrated ${ }^{12} \mathrm{CO}$ and ${ }^{13} \mathrm{CO} J=1 \rightarrow 0$ maps in Fig. 1 show significant emission for $\Delta \delta \lesssim 22$ '4, the position referred to as the cloud edge. In the smoothed ${ }^{12} \mathrm{CO}$ spectra, emission is also evident in the halo (Figs. 2-4). For these positions, even ${ }^{13} \mathrm{CO} J=1 \rightarrow 0$ emission is detected. The higher

6 Practically, the accuracy of spectra with a large signal-to-noise ratio $(S / N \gtrsim 10)$ is dominated by the systematic errors. This category essentially includes all $\mathrm{CO}$ spectra except those with offsets $\Delta \delta \gtrsim 22$ '.4. The accuracy of the [CI] line intensity and the CO intensity in the spectra that show weak or no emission is dominated by the statistical noise. In particular, the systematic error of the [CI] line intensity is small owing to the large main-beam efficiency of the SWAS telescope. 

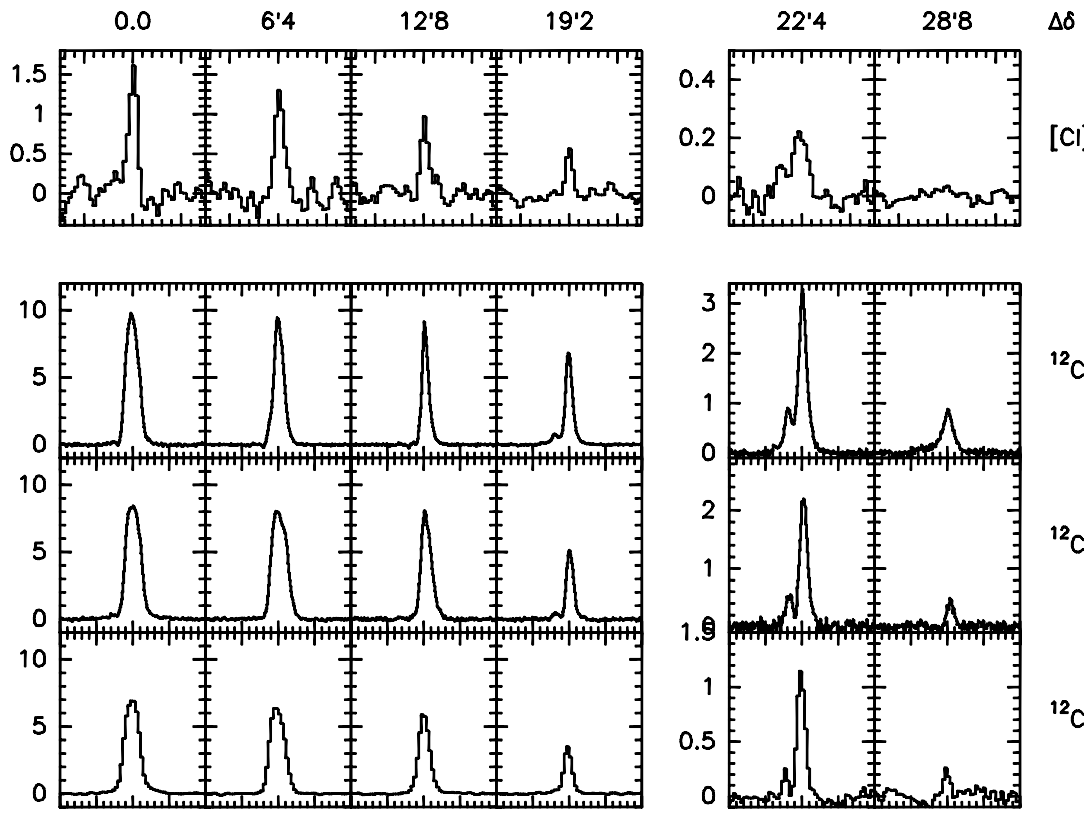

${ }^{12} \operatorname{co}(1-0)$
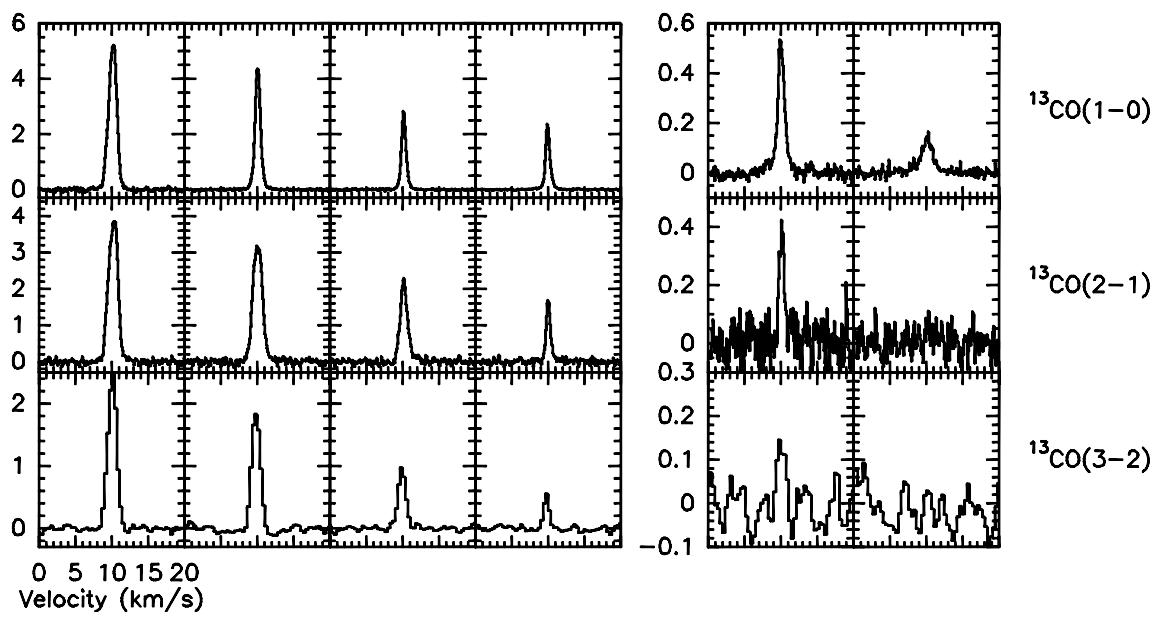

Fig. 2. $[\mathrm{CI}],{ }^{12} \mathrm{CO}$, and ${ }^{13} \mathrm{CO}$ spectral line observations toward selected positions on the N-S strip at $\alpha=3^{\mathrm{h}} 47^{\mathrm{m}} 41^{\mathrm{s}} 6$. Each column includes the spectra toward a single position. The $\Delta \delta$ is indicated at the top of each column and gives the offset from $\delta=32^{\circ} 51^{\prime} 13^{\prime \prime}$, and $\Delta \delta=0^{\prime}$ is the position closest to the line-of-sight toward IRS 1 in the B5 core. Each horizontal row comprises the observations of one transition, indicated on the right hand side of the plot. The ${ }^{12} \mathrm{CO}$ and ${ }^{13} \mathrm{CO}$ spectra are smoothed to the angular resolution of the SWAS [CI] ${ }^{3} \mathrm{P}_{1} \rightarrow{ }^{3} \mathrm{P}_{0}$ data. The temperature scale is $T_{\mathrm{mb}}$, except for the ${ }^{12} \mathrm{CO}$ and ${ }^{13} \mathrm{CO} J=1 \rightarrow 0$ data, which are scaled to the corrected main beam temperature, $T_{\mathrm{mb}, \mathrm{c}}$ (see Sect. 3.1).

${ }^{13} \mathrm{CO}$ transitions, in contrast, are only detected for $\Delta \delta \lesssim 25.6$ (for ${ }^{13} \mathrm{CO} J=2 \rightarrow 1$ ) and $\Delta \delta \lesssim 22: 4\left({ }^{13} \mathrm{CO} J=3 \rightarrow 2\right.$ ). Similarly, $[\mathrm{CI}]$ emission is only detected for $\Delta \delta \lesssim 22$ '.4, despite the longer integration time and thus higher sensitivity of the observations toward the halo. No [CI] emission is visible even for a spectrum where all SWAS observations toward the halo region have been co-added, down to a $3 \sigma$ limit of $40 \mathrm{mK}$ per velocity channel. This spectrum corresponds to an integration time of more than $40 \mathrm{~h}$ with SWAS.

The ${ }^{12} \mathrm{CO}$ and ${ }^{13} \mathrm{CO} \quad J=1 \rightarrow 0$ emission is detected throughout the halo region and up to $\Delta \delta=44$. 8 , including the line-of-sight toward HD 23625. The ${ }^{12} \mathrm{CO}$ line profile of this spectrum suggests the presence of at least two velocity components (Fig. 3). The peak of the stronger component is at $v=10.0 \pm 0.1 \mathrm{~km} \mathrm{~s}^{-1}$, thus at the same $v_{\text {lsr }}$ as the B5 molecular cloud, suggesting that the detected emission traces $\mathrm{CO}$ gas associated with B5. This emission cannot be attributed solely to a possible error-beam pick-up from the main cloud. The line profile shows significant variation for positions separated by only $\sim 3^{\prime}$, and thus at an angular separation that is much smaller than the distance to the B5 molecular cloud and the likely angular extent of the error beam pattern. On the other hand, it cannot be excluded that the $\mathrm{CO}$ emission is even stronger toward these positions because of a possible contamination of the second reference position. This reference position was judged to be emission-free solely on the basis of the present data showing no "absorption" dip below the zero level of the baseline.

The second velocity component visible in Fig. 3 peaks at $v \approx 6.0 \mathrm{~km} \mathrm{~s}^{-1}$. It is plausible that it traces an extension of the Taurus molecular cloud. The $\mathrm{CO}$ emission of the Taurus dark clouds to the South-East of B5 (L1484, 
Table 2. Peak temperature $T_{\mathrm{mb}}(\mathrm{K})$ and results of the escape probability model.

\begin{tabular}{|c|c|c|c|c|c|c|c|c|c|c|}
\hline \multirow[b]{2}{*}{$\Delta \delta^{a}$} & \multirow{2}{*}{$\begin{array}{c}{[\mathrm{CI}]} \\
{ }^{3} \mathrm{P}_{1} \rightarrow{ }^{3} \mathrm{P}_{0}\end{array}$} & \multicolumn{3}{|c|}{${ }^{12} \mathrm{CO}$} & \multicolumn{3}{|c|}{${ }^{13} \mathrm{CO}$} & \multicolumn{3}{|c|}{ Escape probability model } \\
\hline & & $J=1 \rightarrow 0$ & $J=2 \rightarrow 1$ & $J=3 \rightarrow 2$ & $J=1 \rightarrow 0$ & $J=2 \rightarrow 1$ & $J=3 \rightarrow 2$ & $T_{\text {kin }}(\mathrm{K})$ & $\log (n)$ & $\log \left(\mathrm{d} N_{\mathrm{CO}} / \mathrm{d} v\right)$ \\
\hline 0.0 & $1.6 \pm 0.2$ & $9.6 \pm 3.7$ & $8.2 \pm 1.0$ & $6.8 \pm 0.8$ & $5.5 \pm 1.9$ & $3.8 \pm 0.5$ & $2.4 \pm 0.3$ & $13 \pm 1$ & $\geq 4.0$ & $17.5 \pm 0.3$ \\
\hline 3.2 & $1.2 \pm 0.2$ & $9.1 \pm 3.5$ & $8.1 \pm 1.0$ & $6.5 \pm 0.8$ & $5.2 \pm 1.8$ & $3.6 \pm 0.4$ & $2.4 \pm 0.3$ & $13 \pm 1$ & $\geq 4.0$ & $17.5 \pm 0.5$ \\
\hline 6.4 & $1.2 \pm 0.2$ & $9.3 \pm 3.5$ & $8.0 \pm 1.0$ & $6.3 \pm 0.8$ & $4.3 \pm 1.6$ & $3.0 \pm 0.4$ & $1.7 \pm 0.2$ & $13.5 \pm 1$ & $>2.0$ & $17.0-19.0$ \\
\hline 9.6 & $0.78 \pm 0.19$ & $9.2 \pm 3.5$ & $8.0 \pm 1.0$ & $6.0 \pm 0.7$ & $3.5 \pm 1.2$ & $2.3 \pm 0.3$ & $1.1 \pm 0.13$ & $13 \pm 1$ & $4.5 \pm 0.5$ & $17.0 \pm 0.5$ \\
\hline 12.8 & $0.95 \pm 0.15$ & $9.0 \pm 3.4$ & $8.0 \pm 1.0$ & $5.8 \pm 0.7$ & $3.0 \pm 1.0$ & $2.3 \pm 0.3$ & $0.9 \pm 0.11$ & $12.5 \pm 1$ & $4.3 \pm 0.7$ & $17.0_{-0.3}^{+0.5}$ \\
\hline 16.0 & $0.90 \pm 0.14$ & $9.4 \pm 3.6$ & $7.7 \pm 1.0$ & $5.5 \pm 0.7$ & $4.3 \pm 1.5$ & $2.9 \pm 0.4$ & $1.1 \pm 0.13$ & $12.5 \pm 1$ & $3.0_{-1.0}^{0.5}$ & $18.0 \pm 0.5$ \\
\hline 19.2 & $0.56 \pm 0.10$ & $6.5 \pm 2.5$ & $5.0 \pm 0.6$ & $3.4 \pm 0.4$ & $2.5 \pm 0.86$ & $1.6 \pm 0.2$ & $0.5 \pm 0.06$ & $10 \pm 1$ & $<5.0$ & $17.7 \pm 0.7$ \\
\hline 22.4 & $0.20 \pm 0.04$ & $3.2 \pm 1.2$ & $2.2 \pm 0.3$ & $1.1 \pm 0.2$ & $0.53 \pm 0.18$ & $0.40 \pm 0.08$ & $0.13 \pm 0.04$ & $-^{b}$ & $-^{b}$ & $-{ }^{b}$ \\
\hline 25.6 & $<0.054$ & $1.6 \pm 0.6$ & $0.9 \pm 0.15$ & $0.38 \pm 0.06$ & $0.26 \pm 0.09$ & $0.20 \pm 0.05$ & $<0.12$ & & & \\
\hline 28.8 & $<0.069$ & $0.85 \pm 0.3$ & $0.4 \pm 0.1$ & $0.25 \pm 0.07$ & $0.15 \pm 0.05$ & $<0.13$ & $<0.13$ & & & \\
\hline 32.0 & $<0.093$ & $0.5 \pm 0.2$ & $0.2 \pm 0.05$ & $0.10 \pm 0.07$ & $0.13 \pm 0.05$ & $<0.14$ & $<0.14$ & & & \\
\hline
\end{tabular}

a Observations along a N-S strip at $\alpha=3^{\mathrm{h}} 47^{\mathrm{m}} 41^{\mathrm{s}} 6$ (J2000). $\Delta \delta=0.0$ is at $\delta=32^{\circ} 51^{\prime} 13^{\prime \prime}$ (“"( 0,0$)$ position”).

${ }^{b} \chi^{2}$ surface does not show a well-defined minimum.
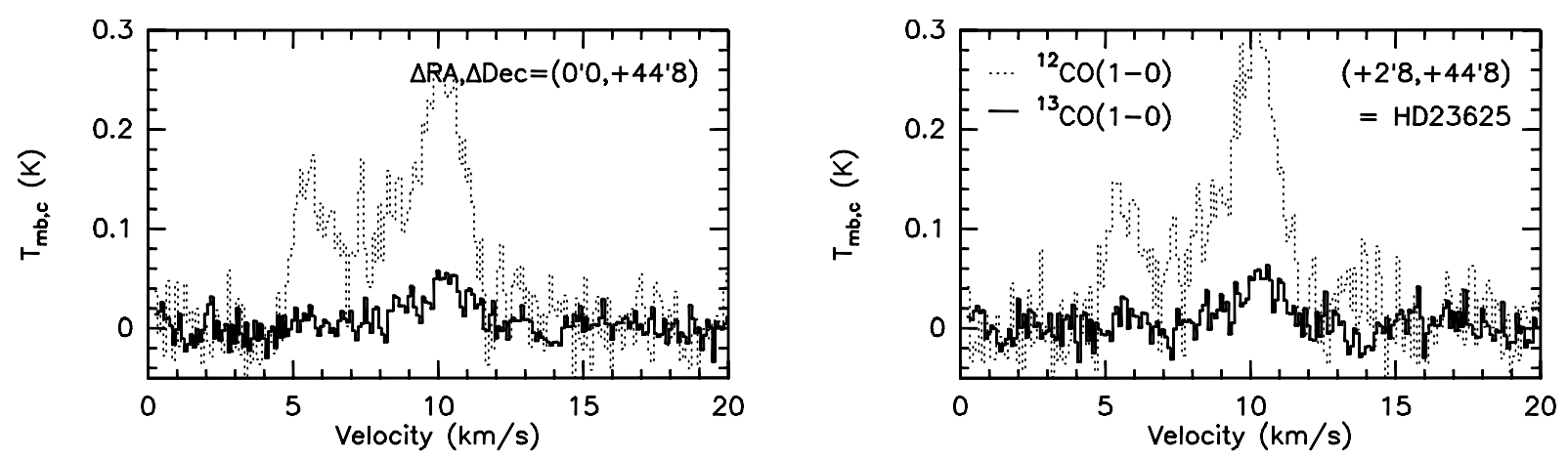

Fig. 3. The ${ }^{12} \mathrm{CO}$ and ${ }^{13} \mathrm{CO} J=1 \rightarrow 0$ emission toward two positions in the B5 halo. The offsets indicated in the top right corner of each panel give the angular distance to the (0,0)-position at $\alpha=3^{\mathrm{h}} 47^{\mathrm{m}} 41^{\mathrm{s}} 6, \delta=32^{\circ} 51^{\prime} 13^{\prime \prime}$. The panel on the right shows the spectra for the line-of-sight toward HD 23625, one of the stars in the absorption-line study by Wannier et al. (1999). The angular resolution of the spectra is 4!3.
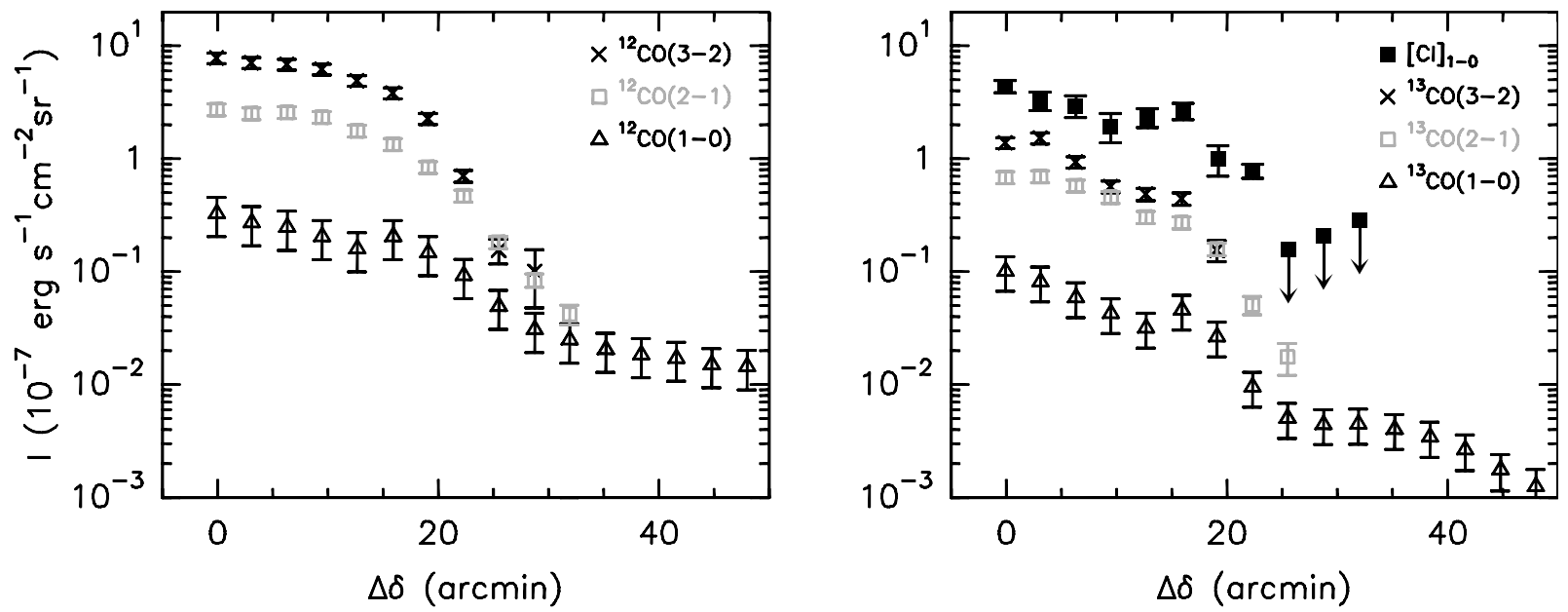

Fig. 4. The $\mathrm{CO},{ }^{13} \mathrm{CO}$, and $[\mathrm{CI}]{ }^{3} \mathrm{P}_{1} \rightarrow{ }^{3} \mathrm{P}_{0}$ line-integrated intensity along the cut outlined in the right panel of Fig. 1, in units of $10^{-7} \mathrm{erg} \mathrm{s}^{-1} \mathrm{~cm}^{-2} \mathrm{sr}^{-1}$. The transitions are indicated in the top right corner of both panels. The three dark squares with the downward-pointing arrows give $3 \sigma$ upper limits. For $\Delta \delta>32^{\prime}$, only observations of the ground-state transition of ${ }^{12} \mathrm{CO}$ and ${ }^{13} \mathrm{CO}$ are available, because the corresponding maps extend to higher declinations.

L1495) is at $v_{\mathrm{lsr}} \sim 6.9 \mathrm{~km} \mathrm{~s}^{-1}$ (Ungerechts \& Thaddeus 1987), close to the velocity of the secondary peak in the ${ }^{12} \mathrm{CO}$ halo spectra. In addition, CO-absorption studies presented by
Kaczmarczyk (2000) suggest two absorbing layers for stars toward the South-East of B5, one of which is attributed to an extension of the Taurus cloud complex. 

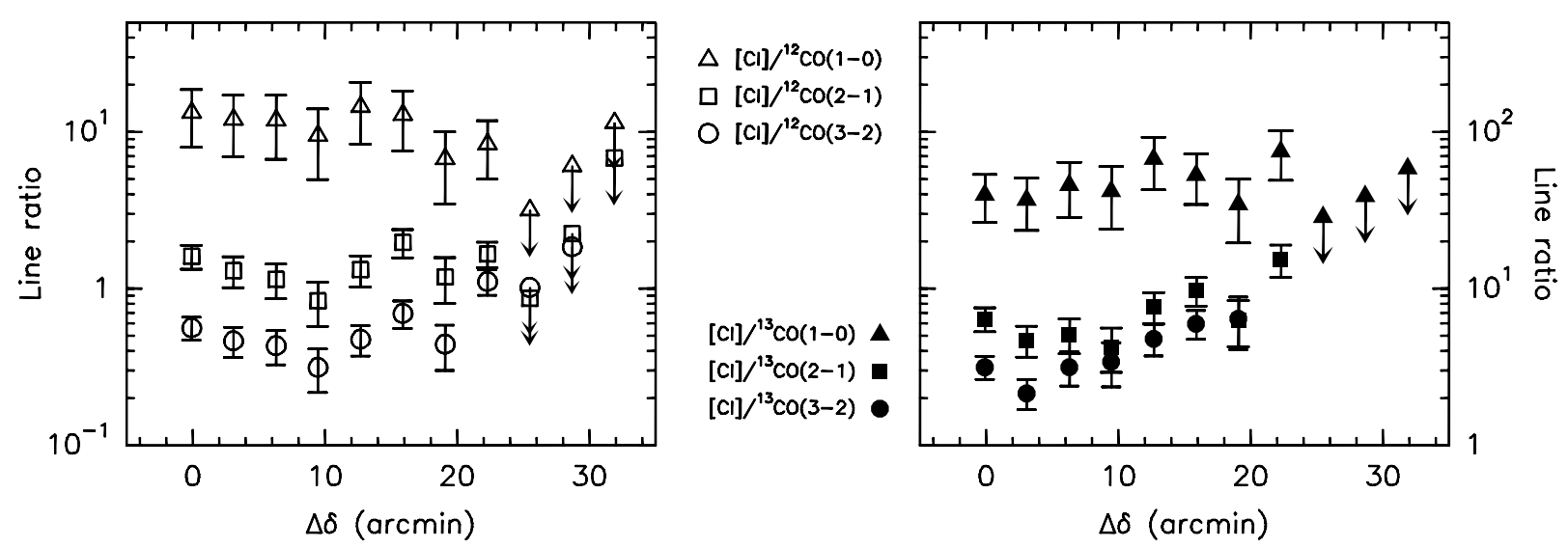

Fig. 5. Left panel: $[\mathrm{CI}] /{ }^{12} \mathrm{CO}$ line-integrated intensity ratio versus offset position. (Line intensity in units of erg $\mathrm{cm}^{-2} \mathrm{~s}^{-1} \mathrm{sr}^{-1}$.) The corresponding $[\mathrm{CI}] /{ }^{13} \mathrm{CO}$ line ratios are shown in the right panel. The error bars give the accuracy of the line ratio (see discussion in Sect. 4). Markers with downward-pointing arrows indicate $3 \sigma$ upper limits.

Table 3. Ratio of line-integrated intensities ${ }^{a}$ observed in B5.

\begin{tabular}{|c|c|c|c|c|c|}
\hline \multirow[b]{2}{*}[\mathrm{CI}]{$/ \mathrm{CO}$ line ratio } & \multicolumn{4}{|c|}{ region $^{b}$} & \\
\hline & core & IRS 4 & edge & halo & \\
\hline$[\mathrm{CI}] /{ }^{12} \mathrm{CO} J=1 \rightarrow 0$ & $12.4 \pm 0.6 \pm 5.2$ & $12.3 \pm 2.1 \pm 5.4$ & $8.4 \pm 3.4$ & $<3.2^{c}$ & \\
\hline$[\mathrm{CI}] /{ }^{12} \mathrm{CO} J=2 \rightarrow 1$ & $1.4 \pm 0.2 \pm 0.3$ & $1.4 \pm 0.5 \pm 0.3$ & $1.7 \pm 0.3$ & $<0.9^{c}$ & \\
\hline$[\mathrm{CI}] /{ }^{12} \mathrm{CO} J=3 \rightarrow 2$ & $0.5 \pm 0.1 \pm 0.1$ & $0.5 \pm 0.2 \pm 0.2$ & $1.1 \pm 0.2$ & $<1.1^{c}$ & \\
\hline$[\mathrm{CI}] /{ }^{13} \mathrm{CO} J=1 \rightarrow 0$ & $41 \pm 4 \pm 15$ & $54 \pm 11 \pm 21$ & $75 \pm 26$ & $<20^{c}$ & \\
\hline$[\mathrm{CI}] /{ }^{13} \mathrm{CO} J=2 \rightarrow 1$ & $5.4 \pm 0.7 \pm 1.2$ & $7.2 \pm 2.3 \pm 1.7$ & $15.4 \pm 3.6$ & - & \\
\hline$[\mathrm{CI}] /{ }^{13} \mathrm{CO} J=3 \rightarrow 2$ & $2.8 \pm 0.5 \pm 0.6$ & $4.7 \pm 1.0 \pm 1.2$ & - & - & \\
\hline CO line ratio & core & IRS 4 & edge & halo & $\operatorname{LTE}^{d}(13 \mathrm{~K})$ \\
\hline${ }^{12} \mathrm{CO} J=2 \rightarrow 1 / J=1 \rightarrow 0$ & $9.3 \pm 0.9 \pm 3.7$ & $9.6 \pm 2.2 \pm 3.9$ & $5.0 \pm 2.0$ & $2.7 \pm 0.8 \pm 1.1$ & 6.7 \\
\hline${ }^{12} \mathrm{CO} J=3 \rightarrow 2 / J=2 \rightarrow 1$ & $2.8 \pm 0.1 \pm 0.5$ & $2.8 \pm 0.1 \pm 0.5$ & $1.5 \pm 0.3$ & $1.0 \pm 0.2 \pm 0.5$ & 2.7 \\
\hline${ }^{13} \mathrm{CO} J=2 \rightarrow 1 / J=1 \rightarrow 0$ & $7.7 \pm 1.4 \pm 2.6$ & $8.1 \pm 1.9 \pm 2.7$ & $4.9 \pm 1.8$ & $<3.1^{c}$ & 6.8 \\
\hline${ }^{13} \mathrm{CO} J=3 \rightarrow 2 / J=2 \rightarrow 1$ & $1.9 \pm 0.2 \pm 0.3$ & $1.5 \pm 0.2 \pm 0.3$ & $<1.8^{c}$ & - & 2.7 \\
\hline
\end{tabular}

${ }^{a}$ The table gives the ratio of the line-integrated intensities $I_{1} / I_{2}$, where $I_{1}, I_{2}$ are in units of $\mathrm{erg} \mathrm{cm}^{-2} \mathrm{~s}^{-1} \mathrm{sr}^{-1}$. The first error gives the rms variation of the line ratio in the region, and the second the systematic error resulting from the accuracy of the line-integrated intensity. The exception is the edge position, where only the systematic error is given.

${ }^{b}$ Regions: core $\left(\Delta \delta \leq 66^{\prime} 4\right)$, IRS $4\left(6^{\prime} 4<\Delta \delta \leq 16^{\prime} \cdot 0\right)$, edge $\left(\Delta \delta=22^{\prime} 4\right)$, halo $\left(\Delta \delta \geq 25^{\prime} \cdot 6\right)$.

${ }^{c} 3 \sigma$ upper limit.

${ }^{d}$ Line ratio for optically thick emission, LTE and $T_{\text {kin }}=13 \mathrm{~K}$.

\subsection{Line ratios}

Figure 5 presents the $[\mathrm{CI}] /{ }^{12} \mathrm{CO}$ and $[\mathrm{CI}] /{ }^{13} \mathrm{CO}$ ratios for the line-integrated intensity as a function of offset $\Delta \delta$. The average and rms of the line ratios for the different regions are listed in Table 3. Generally, the $[\mathrm{CI}] / \mathrm{CO}$ line ratios show little variation across the cloud core and the IRS 4 region. The [CI] to ground-state ${ }^{12} \mathrm{CO}\left({ }^{13} \mathrm{CO}\right)$ ratio is rather constant for all positions where $[\mathrm{CI}]$ is detected. For the higher $\mathrm{CO}$ transitions, the $[\mathrm{CI}] / \mathrm{CO}$ line ratio shows a local minimum at $\Delta \delta \sim 9$ '.6 and then gradually increases toward the cloud edge by a factor of $\sim 2$. The exception to this trend is $\Delta \delta \sim 19$ '2, close to the tip of a filament visible in the ${ }^{13} \mathrm{CO}$ map. Here, the line ratios are similar to those found in the core region, but somewhat lower than in the surrounding regions (IRS 4 and cloud edge).

The spatial variation of the $[\mathrm{CI}]$ emission and the $[\mathrm{CI}] / \mathrm{CO}$ line ratios is somewhat surprising if viewed in the context of a photo-chemical model for clouds in the ISRF. The [CI] emission is expected to primarily trace the cloud surface because the neutral carbon abundance peaks at low visual extinctions $\left(A_{\mathrm{v}} \lesssim 3\right)$ and because of the higher gas temperature close to the surface. Thus, an increasing $[\mathrm{CI}] / \mathrm{CO}$ ratio is expected for larger cloud impact parameter, with a possible limb-brightening of the $[\mathrm{CI}]$ emission at the cloud edge. Even though the $[\mathrm{CI}]$ intensity becomes comparable to the intensity emitted in the $\mathrm{CO}$ lines at $\Delta \delta=22 ! 4$, a pronounced peak in the [CI] emission or $[\mathrm{CI}] / \mathrm{CO}$ line ratios is not observed. One might attribute this to the beam dilution in the relatively large SWAS main beam. Nonetheless, it is remarkable that the $[\mathrm{CI}] / \mathrm{CO} J=1 \rightarrow 0$ line ratio stays fairly constant along the N-S cut where the visual extinction decreases from $A_{\mathrm{v}} \gtrsim 7$ toward the core to $A_{\mathrm{v}} \sim 2$ at the cloud edge.

The neutral carbon column density derived by Wannier et al. (1999) from the UV absorption measurements in the halo is typically an order of magnitude larger than those for $\mathrm{CO}$. However, the sub-mm observations fail to detect the [CI] emission in the halo, while the $\mathrm{CO}$ rotational lines are detected for all positions observed. The $3 \sigma$ upper limit to the $[\mathrm{CI}] / \mathrm{CO}$ 
ratio in the halo are about the same as or even lower than those derived for the molecular cloud.

The carbon column density derived from the UV data varies by between $(1.7-4.8) \times 10^{15} \mathrm{~cm}^{-2}$. For a comparison of the radio emission line data to these UV absorption measurements, one has to consider that the radio data have been smoothed to the relatively large SWAS beam while the UV absorption-line data sample the halo along a pencil beam toward four background stars with a separation of $1-3^{\circ}$. Nonetheless, a $N_{\mathrm{C}^{0}}$ of a few times $10^{15} \mathrm{~cm}^{-2}$ can be considered as typical for the extended halo. Larger column densities are expected toward the SWAS lines-of-sight if we assume a spherical halo, because they are closer to the molecular cloud than probed by the absorption-line data.

\section{Excitation conditions of the gas}

The line excitation of $\mathrm{CO}$ and neutral carbon in the B5 molecular cloud and halo are discussed in this section assuming homogeneous excitation conditions. This gives a first estimate on the physical conditions and the gas column densities in the different regions.

\subsection{Molecular cloud}

The emission of all three ${ }^{12} \mathrm{CO}$ rotational transition observed toward the core and IRS 4 region $\left(\Delta \delta \leq 166^{\prime} 0\right)$ is consistent with LTE, $T_{\text {kin }}=13 \pm 1 \mathrm{~K}$, and a beam filling factor of unity. The peak temperatures listed in Table 2 consistently give a lower limit of $\sim 13 \mathrm{~K}$, if corrected for the Rayleigh-Jeans approximation. Also, the ${ }^{12} \mathrm{CO}$ line ratios in the cloud core and IRS 4 region are consistent with LTE at $T_{\text {kin }}=13 \mathrm{~K}$ and with optically thick emission (compare the last column in Table 3 ). The ${ }^{12} \mathrm{CO} J=2 \rightarrow 1 / J=1 \rightarrow 0$ ratio appears to be systematically higher than expected for optically thick emission, but is still consistent within the relatively large errors bars. It is plausible that the (approximate) error-beam correction discussed in Sect. 3 slightly underestimates the ${ }^{12} \mathrm{CO} J=1 \rightarrow 0$ line intensity, since the kinetic temperature derived from the ${ }^{12} \mathrm{CO}$ $J=1 \rightarrow 0$ peak temperatures is consistently smaller than the temperature derived from the higher ${ }^{12} \mathrm{CO}$ transitions.

Even the ${ }^{13} \mathrm{CO} J=2 \rightarrow 1 / J=1 \rightarrow 0$ ratio observed in the cloud core and the IRS 4 region agrees with the ratio expected for optically thick emission and LTE. This indicates large line opacities even for the lowest two transitions of the rarer isotopologue. Line ratios that are significantly smaller than LTE are noted only for ${ }^{13} \mathrm{CO} J=3 \rightarrow 2 / J=2 \rightarrow 1$. A systematic decrease is observed for all CO line ratios from the IRS 4 region to the edge and the halo region, suggesting that the line excitation becomes sub-thermal for the higher rotational transition at $\Delta \delta \gtrsim 16$ '.0. A reduced optical depth of the higher rotational transition might also contribute. Non-LTE effects, however, are likely to play the greater role for the $\mathrm{CO}$ emission close to the cloud edge. For small column densities (optical depths), the CO line ratios are expected to be higher than in the optically thick case. The fact that the ${ }^{12} \mathrm{CO}$ line ratios are below the optically thick/LTE case and consistent with the ${ }^{13} \mathrm{CO}$-line ratios points
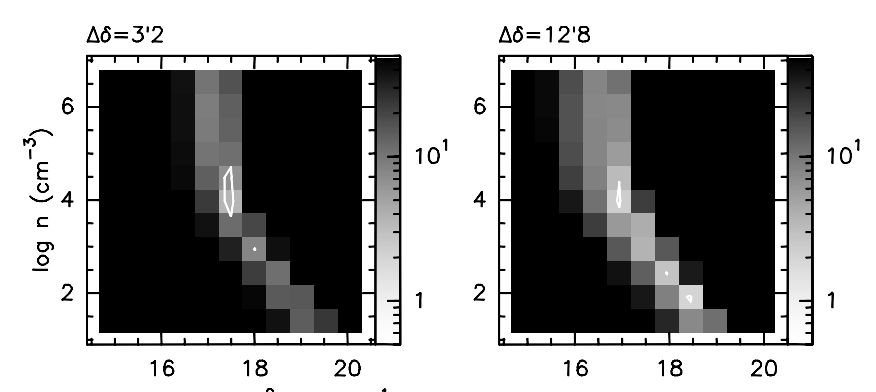

$\log \mathrm{dN}_{\mathrm{co}} / \mathrm{dv}\left(\mathrm{cm}^{-2}(\mathrm{~km} / \mathrm{s})^{-1}\right)$

Fig. 6. $\chi^{2}$ surface for the escape probability model and the observations toward two positions on the N-S cut. The offset on the cut is indicated at the top of the panels. The plots show the reduced $\chi^{2}$ as a function of $\log (n)$ and $\log \left(\mathrm{d} N_{\mathrm{CO}} / \mathrm{d} v\right)$ for models with a constant temperature of $T_{\text {kin }}=13 \mathrm{~K}$. Contours outline $\chi^{2}=3 \times \chi_{\min }^{2}$, where $\chi_{\min }^{2}$ is the minimum in each panel. Similar plots with different temperatures show that the $\chi^{2}$ steeply rises for $T<12 \mathrm{~K}$ and $T>14 \mathrm{~K}$.

to a sub-thermal excitation rather than optical depth effects as the cause for the lower line ratios at the cloud edge.

A more detailed and quantitative analysis of the $\mathrm{CO}$ observations in the molecular cloud is done by using the escape probability code by Stutzki \& Winnewisser (1984). This code accounts for non-LTE excitation and the effects from finite optical depths. The observations in the halo are discussed in Sect. 5.2 because the line excitation is quite different from the one in the molecular cloud. The escape probability model calculates the line temperature (in $\mathrm{K}$ ) for the ${ }^{12} \mathrm{CO}$ and ${ }^{13} \mathrm{CO}$ rotational transitions as a function of the free parameter gas temperature, density, and $\mathrm{CO}$ column density per velocity interval $\mathrm{d} N_{\mathrm{CO}} / \mathrm{d} v$. The results are compared to the observed peak temperatures listed in Table 2 for the core region, IRS 4 region, and the cloud edge. The parameter space covered by the models is $10 \mathrm{~K} \leq T \leq 60 \mathrm{~K}, 1.5 \leq \log n \leq 6.5$, and $15.0 \leq \log \left(\mathrm{d} N_{\mathrm{CO}} / \mathrm{d} v\right) \leq 20.0$ for ${ }^{12} \mathrm{CO}$, and an abundance ratio of ${ }^{12} \mathrm{CO} /{ }^{13} \mathrm{CO}=65$ is assumed. For each model, the reduced $\chi^{2}=m^{-1} \sum_{i}^{m}\left(T_{\mathrm{i}, \text { obs }}-T_{\mathrm{i}, \mathrm{mod}}\right)^{2} / \Delta T^{2}$ is calculated, where $T_{\mathrm{i}, \text { obs }}$ is the observed peak temperature, $T_{\mathrm{i} \text {,mod }}$ the model result, and $\Delta T$ gives the accuracy of the observed peak temperatures. The sum is over the observed transitions, which are the three lowest ${ }^{12} \mathrm{CO}$ and ${ }^{13} \mathrm{CO}$ transitions. The resulting $\chi^{2}$ is plotted as a function of the model parameters, and the minimum of the $\chi^{2}$ function gives the best-fit model parameter. An example is shown in Fig. 6 for the peak temperatures observed toward two positions in the cloud. The results for the eight positions with $\Delta \delta \leq 22$ '. 4 are summarized in Table 2 .

The escape probability model gives typical gas kinetic temperatures of $T_{\text {kin }}=12-13 \mathrm{~K}$ for the position in the core and IRS 4 region, in agreement with the LTE discussion above and the results by Young et al. (1982) and Langer et al. (1989). Note, that the higher gas temperatures of $T>25 \mathrm{~K}$ derived by Young et al. (1982) for the cloud edge correspond to larger offsets from the cloud center $\left(\Delta \delta \gtrsim 20^{\prime}\right.$ relative to the " $(0,0)$ position" used in the present paper). Typical gas densities derived in the present study for the core and most of the IRS 4 region $\left(\Delta \delta \lesssim 12\right.$.8) are $n \gtrsim 10^{4} \mathrm{~cm}^{-3}$, an order of magnitude larger than in Young et al. (1982), and even larger than those obtained from the $\mathrm{C}^{18} \mathrm{O}$ observations by 
Goldsmith et al. (1986). This has two possible explanations. First, the peak temperature is used for the present study, presumably probing gas with a higher density than traced by the line-integrated intensity. Second, the higher density reflects the fact that only lower limits can be derived from spectral line observations of gas with LTE excitation conditions, with the lower limit given by the critical density of the highest transition considered. The higher density obtained here can therefore be attributed to the $\mathrm{CO} J=3 \rightarrow 2$ transition $\left(n_{\text {crit }} \sim 5 \times 10^{4} \mathrm{~cm}^{-3}\right)$ included in the present study, whereas Young et al. (1982) use the two lowest CO transitions. We derive a gas density that is significantly below the critical density of the $\mathrm{CO} J=3 \rightarrow 2$ transition for positions with $\Delta \delta \geq 16$ '.0 only (Table 2), and the density for these positions is consistent with the results by Young et al. (1982).

Summarizing, we find that the gas in the core region is consistent with LTE for all ${ }^{12} \mathrm{CO}$ transitions observed. For the IRS 4 region, the ${ }^{12} \mathrm{CO} J=3 \rightarrow 2$ transition is slightly subthermal, but still close to LTE due to a possible contribution from line trapping. The typical density for the IRS 4 region is $n \sim 2 \times 10^{4} \mathrm{~cm}^{-3}$, with higher densities in the core region and lower densities likely to apply close to the cloud edge.

\subsection{Cloud halo}

The CO-line excitation in the halo is dominated by the radiation from the nearby molecular cloud. This can be shown by comparing the line excitation by radiation to the collisional excitation rate using the physical parameters of the neutral halo derived by Wannier et al. (1999), $T \sim 40 \mathrm{~K}$ and $n \sim 70 \mathrm{~cm}^{-3}$. The excitation rate of a $\mathrm{CO}$ molecule by absorption of a line photon emitted by the B5 molecular cloud is

$B_{\mathrm{lu}} J\left(v_{l}\right)=\frac{\Omega_{\mathrm{B} 5}}{4 \pi} \frac{g_{\mathrm{u}}}{g_{\mathrm{l}}} A_{\mathrm{ul}}\left[\exp \left(h v_{l} / k T_{\mathrm{rad}}\right)-1\right]^{-1}$,

where $B_{\text {lu }}$ is the Einstein coefficient for absorption $l \rightarrow u$, and $J\left(v_{l}\right)$ the radiation field energy density at the line frequency. On the right hand side, $g_{\mathrm{u}}$ and $g_{\mathrm{l}}$ are the upper and lower level statistical weights, and $A_{\mathrm{ul}}$ denotes the Einstein coefficient for spontaneous emission, while $\Omega_{\mathrm{B} 5}$ is the solid angle subtended by the B5 cloud, viewed from the halo. The energy density of the radiation field close to the line frequency is approximated by a blackbody of temperature of $T_{\text {rad }}=13 \mathrm{~K}$, the LTE excitation temperature of $\mathrm{CO}$. This is reasonable because the velocity of the $\mathrm{CO}$ molecules in the halo is close to the peak of the $\mathrm{CO}$ emission in B5, with a velocity dispersion the same as or smaller than the line-widths measured toward the B5 cloud.

For the $\mathrm{CO} J=0 \rightarrow 1$ transition and a solid angle of $\Omega_{\mathrm{B} 5} /(4 \pi)=0.15-0.37$, we obtain $B_{\mathrm{lu}} J\left(v_{l}\right)=(0.6-1.5) \times$ $10^{-7} \mathrm{~s}^{-1}$. For comparison, the excitation rate coefficient for collisions with $\mathrm{H}$, the dominant collision partner in the halo, is $1.5 \times 10^{-11} \mathrm{~s}^{-1} \mathrm{~cm}^{3}$ for $T=40 \mathrm{~K}$ (see Green \& Thaddeus 1976, Warin, Benayoun, \& Viala 1996). The collisional excitation rate for $\mathrm{CO} J=0 \rightarrow 1$ in $70 \mathrm{~cm}^{-3}$ density gas is $C_{\mathrm{lu}}=$ $1.0 \times 10^{-9} \mathrm{~s}^{-1}$. This gives a ratio of the radiation excitation over the collisional excitation of $B_{\mathrm{lu}} J\left(v_{l}\right) / C_{\mathrm{lu}}=60-150$ for a solid angle of $\Omega_{\mathrm{B} 5} /(4 \pi)=0.15-0.37$. A similar ratio $\left(\gtrsim 10^{2}\right)$ is obtained for the higher rotational levels $J=2,3$. The conclusion does not change when additional collisions with $\Delta J>1$ are included, and if $\mathrm{H}_{2}$ is considered as a collision partner rather than $\mathrm{H}$ (the collision rate of $\mathrm{CO}$ with $\mathrm{H}_{2}$ is larger by a factor of $\sim 8$ only, see Flower \& Launay 1985). Thus, line absorption is the dominant process, even if molecular hydrogen were the most abundant gas species in the halo rather than $\mathrm{H}$. The radiative excitation rate is smaller than the coefficient for spontaneous emission, however, and the excitation temperature of $\mathrm{CO}$ in the halo is not adjusted to the $13 \mathrm{~K}$ radiation temperature of the B5 cloud. This is consistent with the studies by Wannier, Penprase \& Andersson (1997), which demonstrate that the rotational excitation of $\mathrm{CO}$ in diffuse, low-density gas $\left(n \lesssim 300 \mathrm{~cm}^{-3}\right)$ in the vicinity of dense interstellar clouds is dominated by the absorption of line photons from the denser material.

For neutral carbon, in contrast, the line excitation is dominated by collisions and not by [CI] line photons emitted by the nearby molecular cloud. The radiative excitation scales with the molecular parameter and the radiation temperature as $B_{\text {lu }} J\left(v_{1}\right) \propto\left(g_{\mathrm{u}} / g_{1}\right) A_{\mathrm{ul}}\left[\exp \left(h v_{l} / k T_{\mathrm{rad}}\right)-1\right]^{-1}$. The term $\left(g_{\mathrm{u}} / g_{1}\right) A_{\mathrm{ul}}$ is about the same for CO $J=1 \rightarrow 0$ and [CI] ${ }^{3} \mathrm{P}_{1} \rightarrow{ }^{3} \mathrm{P}_{0}$. The denominator in the exponential term, however, is much larger for $[\mathrm{CI}]$ because the transition energy (in $\mathrm{K}$ ) is much larger than the radiation temperature of the line emission, $h v_{l} / k T_{\text {rad }} \gg 1$. Thus, the radiative excitation rate for the ground-state $[\mathrm{CI}]$ transition is between $(2.9-7.1) \times 10^{-9} \mathrm{~s}^{-1}$ for $\Omega_{\mathrm{B} 5} /(4 \pi)=0.15-0.37$, a factor of $\sim 20$ smaller than for ${ }^{12} \mathrm{CO} J=0 \rightarrow 1$. The collisional excitation rate, in contrast, is more than an order of magnitude larger for [CI] than for the $\mathrm{CO}$ transitions (again, for collisions of $\mathrm{C}^{0}$ with $\mathrm{H}$; Launay \& Roueff 1977; Warin et al. 1996). The ratio of the radiative to collisional excitation rate, therefore, is smaller than unity, $B_{\text {lu }} J\left(v_{l}\right) / C_{\text {lu }}=0.15-0.37$, indicating that the excitation of the ${ }^{3} P_{1}$ level of neutral carbon is governed by collisions. The critical density of the $[\mathrm{CI}]{ }^{3} \mathrm{P}_{1} \rightarrow{ }^{3} \mathrm{P}_{0}$ transition for collisions with $\mathrm{H}$ is $\sim 500 \mathrm{~cm}^{-3}$, larger than the gas density in the halo. Thus, the $[\mathrm{CI}]$ excitation is expected to be highly sub-thermal.

The escape probability model is employed to derive an upper limit for the neutral carbon column density, based on the rms noise in the $[\mathrm{CI}]$ spectra made toward the halo region. (Recall that no [CI] emission is detected toward the halo at $\Delta \delta \gtrsim 25$ ' 6 , see Fig. 2 and Table 2). The [CI] line temperature is calculated for a grid of models with the kinetic temperature between 20 and $60 \mathrm{~K}$ and the density between $10-1000 \mathrm{~cm}^{-3}$. The neutral carbon column density per velocity interval, $\mathrm{d} N_{\mathrm{C}^{0}} / \mathrm{d} v$, is varied between $10^{14}$ and $10^{17} \mathrm{~cm}^{-2}(\mathrm{~km} / \mathrm{s})^{-1}$. As an example, the $[\mathrm{CI}]$ peak temperature is shown in Fig. 7 as a function of pressure $(n T)$ and $\mathrm{d} N_{\mathrm{C}^{0}} / \mathrm{d} v$ for models with $T=40 \mathrm{~K}$. In addition, the pressure derived by Wannier et al. (1999) for the B5 halo is marked in the plot $\left(n T=2200 \pm 300 \mathrm{~K} \mathrm{~cm}^{-3}\right)$, as well as the $3 \sigma$ upper limit to the peak temperature of the emission derived from the individual SWAS spectra made in the halo $\left(T_{\mathrm{mb}}<63 \mathrm{mK}(\mathrm{km} / \mathrm{s})^{-1}\right)$ and for the co-added spectra in the halo region $\left(T_{\mathrm{mb}}<46 \mathrm{mK}(\mathrm{km} / \mathrm{s})^{-1}\right)$.

Assuming a line width for the $[\mathrm{CI}]$ emission in the halo region similar to the ${ }^{12} \mathrm{CO}$ line width $\left(\sim 2 \mathrm{~km} \mathrm{~s}^{-1}\right)$, we get an upper limit to the total neutral carbon column density of $N_{\mathrm{C}} \lesssim 8 \times 10^{15} \mathrm{~cm}^{-2}\left(N_{\mathrm{C}} \lesssim 6 \times 10^{15} \mathrm{~cm}^{-2}\right.$, respectively). Similar results are obtained for models with $T_{\text {kin }}=60 \mathrm{~K}$, while the 


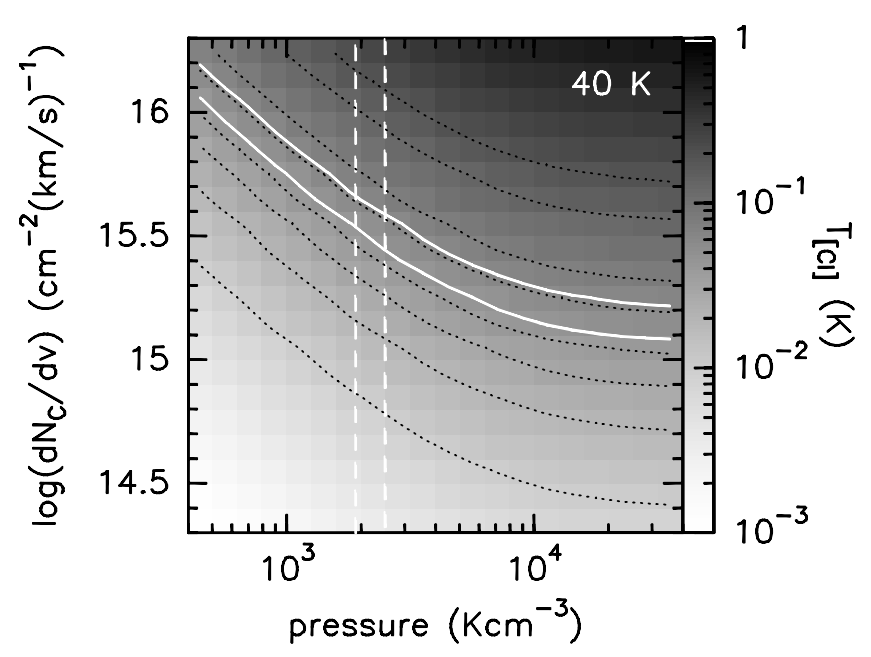

Fig. 7. Escape probability model for $[\mathrm{CI}]{ }^{3} \mathrm{P}_{1} \rightarrow{ }^{3} \mathrm{P}_{0}$ emission in the $\mathrm{B} 5$ cloud halo. The $[\mathrm{CI}]$ peak temperature (in Kelvin) is shown as a function of gas pressure $\left(n T_{\text {kin }}\right)$ and neutral carbon column density per velocity interval $\left(\mathrm{d} N_{\mathrm{C}} / \mathrm{d} v\right)$ for models with $T_{\text {kin }}=40 \mathrm{~K}$. Dark contours are drawn for $10,20,30,40,60,80,140$, and $200 \mathrm{mK}$. The white (solid) contours give the $3 \sigma$ upper limit from the SWAS observations for a single position in the halo $\left(63 \mathrm{mK}\right.$ per $\left.\mathrm{km} \mathrm{s}^{-1}\right)$ and from the coadded spectra in the halo region $\left(46 \mathrm{mK}_{\text {per }} \mathrm{km} \mathrm{s}^{-1}\right)$. The vertical lines indicate the pressure $2200 \pm 300 \mathrm{~K} \mathrm{~cm}^{-3}$ derived by Wannier et al. (1999) for the B5 cloud halo.

upper limits derived from the $20 \mathrm{~K}$ models are larger by $\sim 20 \%$. For comparison, Wannier et al. (1999) derive a neutral carbon column density between $(1.7-4.8) \times 10^{15} \mathrm{~cm}^{-2}$ from the (not velocity-resolved) absorption-line measurements. Thus, the upper limits to the $[\mathrm{CI}]$ emission from the SWAS observations do not rule out neutral carbon column densities of the order of those derived from the UV absorption-line data. However, they rule out that the neutral carbon column density is significantly higher in the halo close to the B5 cloud (angular distances of ऽfew tens of arcmin) than those probed by the absorption-line study at larger angular distances $\left(\sim 0.5^{\circ}-1.5^{\circ}\right)$. This is plausible if we assume that the halo is not homogeneous and/or spherically symmetric.

A $3 \sigma$ detection of the [CI] emission in the halo with $N_{\mathrm{C}^{0}}=$ $3 \times 10^{15} \mathrm{~cm}^{-2}$ and with a line-width of $\sim 2 \mathrm{~km} \mathrm{~s}^{-1}$ requires an rms noise of $\sim 7 \mathrm{mK}$. The on-source integration that is required is $t_{\mathrm{on}} \sim 210 \min$ (assuming $\Delta v=0.5 \mathrm{~km} \mathrm{~s}^{-1}$ and a system noise temperature of $T_{\text {sys }}=500 \mathrm{~K}$, which is applicable to observations at very high and dry locations such as the Atacama desert in Chile), and $t_{\mathrm{on}}=7 \mathrm{~min}$ (for HIFI band 1 with $T_{\text {sys }}=90 \mathrm{~K}$; Tielens 2001).

Note, however, that the CO observations indicate that there are two components along the line of sight, while the absorption-line data are not spectrally resolved. The neutral carbon column densities of $(1.7-4.8) \times 10^{15} \mathrm{~cm}^{-2}$ derived by Wannier et al. (1999) therefore have to be considered as upper limits for the B5 halo, since a fraction of it might arise in the second component unrelated to B5 (see Fig. 3). More recent $\mathrm{C}^{0}$ absorption-line measurements by Jenkins \& Tripp (2001) are spectrally resolved. However, the rather low neutral carbon column densities of $\lesssim 1 \times 10^{14} \mathrm{~cm}^{-2}\left(\mathrm{~km} \mathrm{~s}^{-1}\right)^{-1}$ probed in their
Table 4. Model parameter of spherical PDR code.

\begin{tabular}{lll}
\hline \hline \multicolumn{3}{l}{ PDR model parameters } \\
\hline$M_{\mathrm{cl}}$ & $10^{-6} \ldots 100 M_{\odot}$ & clump mass \\
$\langle n\rangle_{\mathrm{cl}}$ & $300 \ldots 3 \times 10^{6} \mathrm{~cm}^{-3}$ & average clump density \\
$n_{\mathrm{s}}$ & $n_{\mathrm{s}} \approx 0.5 \times\langle n\rangle_{\mathrm{cl}}$ & clump surface density ${ }^{a}$ \\
\hline$\zeta_{\mathrm{CR}}$ & $1 \times 10^{-17} \mathrm{~s}^{-1}$ & CR ionization rate \\
$A_{\mathrm{v}}$ & $6.3 \times 10^{-22} \times N_{\mathrm{H}}$ & visual extinction \\
$\tau_{\mathrm{UV}}$ & $3.68 A_{\mathrm{v}}$ & FUV dust attenuation \\
$\mathrm{v}_{\mathrm{b}}$ & $1 \mathrm{~km} \mathrm{~s}^{-1}$ & Doppler width \\
\hline $\mathrm{He}$ & 0.1 & abundances $^{b}$ \\
$\mathrm{C}$ & $1.4 \times 10^{-4}$ & \\
${ }^{13} \mathrm{C}$ & $2.2 \times 10^{-6}$ & \\
$\mathrm{O}$ & $3.0 \times 10^{-4}$ & \\
$\mathrm{~N}$ & $1.0 \times 10^{-4}$ & \\
$\mathrm{~S}$ & $2.8 \times 10^{-5}$ & \\
$\mathrm{Si}$ & $1.7 \times 10^{-6}$ & \\
$\mathrm{Mg}$ & $1.1 \times 10^{-6}$ & \\
$\mathrm{Fe}$ & $1.7 \times 10^{-7}$ & \\
\hline
\end{tabular}

${ }^{a}$ For a (truncated) power-law density profile of $n \propto r^{-1.5}$.

${ }^{b}$ Initial abundance, relative to $\mathrm{H}$ nuclei.

sample of diffuse clouds makes a detection of the fine-structure transition rather difficult.

\section{PDR modeling}

In the following, the observations for the $[\mathrm{CI}]$ and the lowest three rotational transitions of ${ }^{12} \mathrm{CO}$ and ${ }^{13} \mathrm{CO}$ toward the B5 molecular cloud (offsets $\Delta \delta \leq 22$ '.4) are compared to PDR simulations. The goal is to test if the observed emission can be successfully modeled as a PDR in the diffuse ISRF.

\subsection{Spherical PDR model}

The PDR simulations use the code developed by Störzer et al. (1996). This model is based on a single spherical clump irradiated by an external, isotropic FUV radiation field ( $h v=$ $6-13.6 \mathrm{eV})$. The clump has a truncated power-law density profile, $n(r) \propto r^{-1.5}$ for $0.2 \leq r / r_{\mathrm{cl}} \leq 1$ and $n(r)=$ const. for $r / r_{\mathrm{cl}} \leq 0.2$, where $r_{\mathrm{cl}}$ is the radius of the clump. Thus, the clump density profile is specified by two parameters, for example, the clump radius $r_{\mathrm{cl}}$ and the density at the clump surface $n_{\mathrm{s}}=n\left(r_{\mathrm{cl}}\right)$. Alternative parameters are the clump-averaged density $\langle n\rangle_{\mathrm{cl}}$ and total clump mass $M_{\mathrm{cl}}$. The fit by Draine (1978) is used for the specific photon intensity of the external FUV field, multiplied by a scaling factor $\chi$. The initial elemental abundances and other basic model parameters are summarized in Table 4.

The original PDR code has been modified in a few instances. An expanded chemical network was included (Bensch et al. 2003), and the more recent release of the chemical reaction rates adopted for the present simulations (UMIST 99, see Le Teuff et al. 2000). The UMIST 99 rate coefficients are modified for a few reactions. Several of the reactions have 
multiple entries for different temperature regimes, and the rate coefficients applicable to the temperatures typical of weakFUV PDRs are used in the present model. A few reactions have their rates specified only for temperatures above a few hundred Kelvins, and some of them have a negative exponent in the exponential term of the UMIST parameterization $(\gamma<0$ in Eq. (1) of Le Teuff et al. 2000). The reaction rates of the latter are substantially overestimated if they are extrapolated to temperatures below $100 \mathrm{~K}$. This applies to a total of 16 reactions, and the UMIST 95 rates are used in this case.

The CO dissociation is treated by employing the shielding factors published by van Dishoeck \& Black (1988). The PDR code allows for additional shielding by a column density of $\mathrm{H}_{2}$ and $\mathrm{CO}$ in a halo surrounding the clump (pre-shielding). This reduces the dissociation rate of $\mathrm{CO}$ and $\mathrm{H}_{2}$ in the clump but does not reduce the gas heating, in contrast to the general attenuation of the FUV field by dust. The pre-shielding column densities are treated as an additional model parameters and are kept constant during the iterations of the numerical code. While preshielding has been included as optional parameter by Störzer, Stutzki \& Sternberg (1996), many of the previously published studies employing this PDR code set them to zero.

The PDR model provides a self-consistent solution of the chemical structure (abundances of species) and of the temperature (thermal balance of the gas) as a function of radius. The radiation transfer code ONION (Gierens et al. 1992) is then used to calculate the peak and line-integrated intensity of the ${ }^{12} \mathrm{CO}$, ${ }^{13} \mathrm{CO}$ rotational transitions, and the $[\mathrm{CI}],[\mathrm{CII}]$ fine-structure transitions from the abundance and temperature profiles provided by the PDR model. More precisely, the peak and the lineintegrated intensities averaged over the projected area of the clump are calculated for the present simulations (see below).

\subsection{PDR model grid and line ratios}

The PDR code is employed to model the observations smoothed to the SWAS angular resolution of 4.3, the resolution of the $[\mathrm{CI}]$ spectra. The $\mathrm{CO}$ map made with the FCRAO telescope has a higher resolution of 44" and shows significant structure for the emission at angular scales that are smaller than the SWAS beam. Assuming a clumpy cloud structure, we expect that the 4.3 SWAS beam samples the emission from a large number of clumps, where the radius of each clump is smaller than that of the beam at the distance of the cloud.

In addition, we assume that each clump in the ensemble has the same mass and density, and that the global line ratio of the emission emitted by the ensemble can be estimated by using the line ratio of a single clump of that mass and density. In other words, we assume that the line ratio is governed by the emission from clumps of similar mass and density, and we neglect effects resulting from the radiative coupling and shadowing of the clumps. The line ratios are calculated from the line-integrated intensities averaged over the projected clump area, because the line ratios are not very sensitive to the beamfilling factor for clumps smaller than the beam (Fig. 8, panels a and $b$ ). In addition, the peak intensity of the clump-averaged line profile calculated from the PDR model can be compared to observations made in regions where the beam-filling factor is close to unity (Fig. 8, panel c). This is the case for the B5 core region, for example.

The goal of the PDR modeling is to identify the typical mass and density of the clumps that govern the line emission in the different regions of the cloud (Table 3). A grid of $9 \times 9$ models is calculated for this purpose, where the mass and the density of the clump is varied over a wide range: $3 \times 10^{2} \mathrm{~cm}^{-3} \leq$ $\langle n\rangle_{\mathrm{cl}} \leq 3 \times 10^{6} \mathrm{~cm}^{-3}$ and $10^{-6} M_{\odot} \leq M_{\mathrm{cl}} \leq 100 M_{\odot}$, respectively. Other PDR model parameters, such as the strength of the external FUV field, are kept constant in each grid, but are varied for subsequent model grids in order to study their impact on the predicted line intensities. Figure 9 gives the clump radius and the visual extinction averaged over the projected clump area for each clump in the model grid. The figure shows that most of the clumps indeed have a much smaller radius than the projected SWAS beam. The exceptions are a few clumps with high $M_{\mathrm{cl}}$ and low $\langle n\rangle_{\mathrm{cl}}$ in the lower right part of the figure. Such clumps are not strictly ruled out, however, as long as the clump-averaged visual extinction and the line emission in the PDR model do not exceed the observed $A_{\mathrm{v}}$ and intensities for the position considered.

\subsection{Model assumptions and limitations}

The spherical (clumpy) PDR model acknowledges the fact that the small-scale structure in molecular clouds plays an important role for the chemical (PDR) structure and thus the line emission of these clouds. The present model calculations assume that the spatial structure of the clouds is made up of clumps of the same mass and density. An even more realistic model considers a clump ensemble where the clump mass and size follows the mass- and size spectrum observed in many molecular clouds (see Zielinsky et al. 2000, for example). In this context, the present simulation is useful because it provides the typical density and size of the regions that dominate the observed line ratios. This does not exclude the presence of clumps that are smaller or larger than those that match the observed line ratios. However, these clumps cannot dominate the emission if the line ratio of these clumps is significantly different from the observed ratios. With the mass and size of the clumps in the model and the observed line ratios and cloud $A_{\mathrm{v}}$, it is possible to estimate an upper limit to the number of these clumps from the constraint that they do not significantly change the observed line ratios.

Shadowing of the clumps is not expected to play a substantial role, at least for regions where the total column density is low, for example the B5 cloud edge and possibly the IRS 4 region. The FUV photons can penetrate to greater depths than possible in a homogeneous medium because of the clumpy cloud structure and the scattering of FUV photons at dust (for example, Boissé 1990). Multiple scattering of photons in clumpy media is also effective in maintaining an approximately isotropic FUV field deeper in the cloud.

Using the line ratios calculated for a single clump as an approximation of the line ratio of a clump ensemble neglects the radiative coupling between individual clumps. This is justified 
(a)
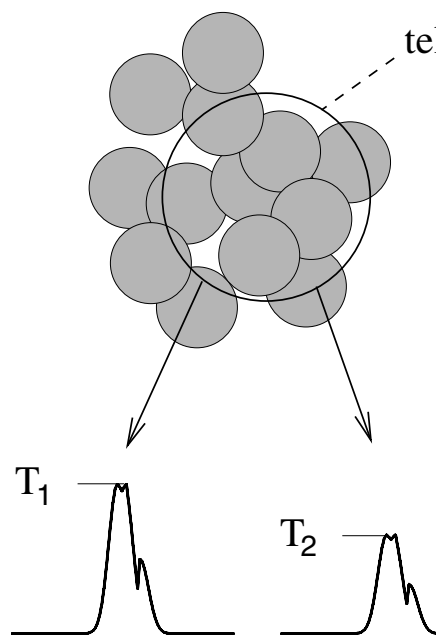

Line 1: $\mathrm{I}_{1}$

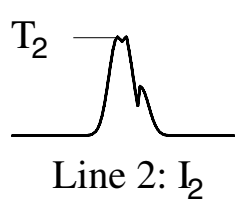

(b)

elescope beam

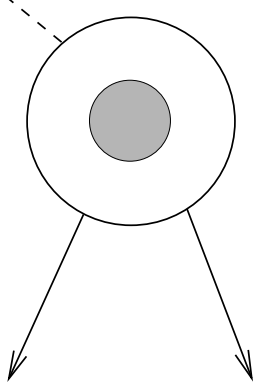

(c)

clump-averaged intensity
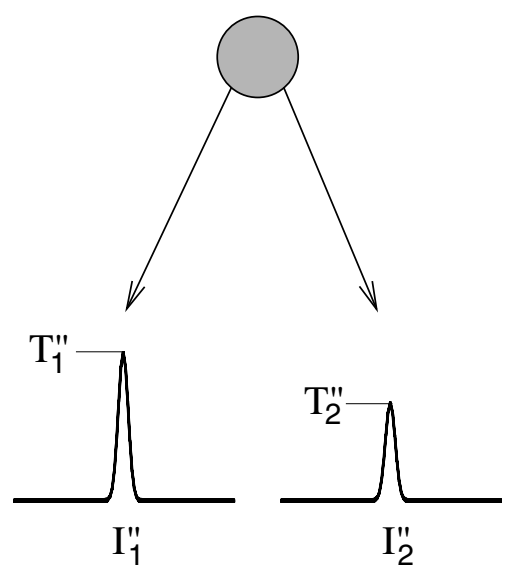

Fig. 8. a) Spectral line observations made for an ensemble of spatially unresolved clumps (clump radius < telescope beam) as an approximation of the spatial structure of molecular clouds. The lower part shows the line profile, integrated intensity $\left(I_{1}, I_{2}\right)$, and the peak temperature $\left(T_{1}, T_{2}\right)$ for two different transitions (line 1,2). Panel b) shows the same situation with only one clump in the beam, and the line-integrated intensities will be different in this case $\left(I_{1}^{\prime} \neq I_{1}, I_{2}^{\prime} \neq I_{2}\right)$. Assuming that the emission by the individual clumps is radiatively decoupled, the ratio of the line-integrated intensity of the clump ensemble can be approximated by the ratio calculated for just one clump: $I_{1} / I_{2} \approx I_{1}^{\prime} / I_{2}^{\prime}$. Panel c) gives the peak and integrated intensity, averaged over the projected area of the clump $\left(T_{1}^{\prime \prime}, T_{2}^{\prime \prime}\right.$ and $I_{1}^{\prime \prime}, I_{2}^{\prime \prime}$, respectively). Again, the line ratio is similar to the ratio in case $\mathbf{a})$ and $\mathbf{b}$ ) where the beam is larger than the projected clump area $\left(I_{1} / I_{2} \approx I_{1}^{\prime \prime} / I_{2}^{\prime \prime}\right)$. In addition, the peak intensity of the emission detected for a clump ensemble is close to the peak intensity of the clump-averaged emission in a single clump, if the beam filling factor of that clump ensemble is close to unity (case a), $T_{1} \approx T_{1}^{\prime \prime}$ and $T_{2} \approx T_{2}^{\prime \prime}$.

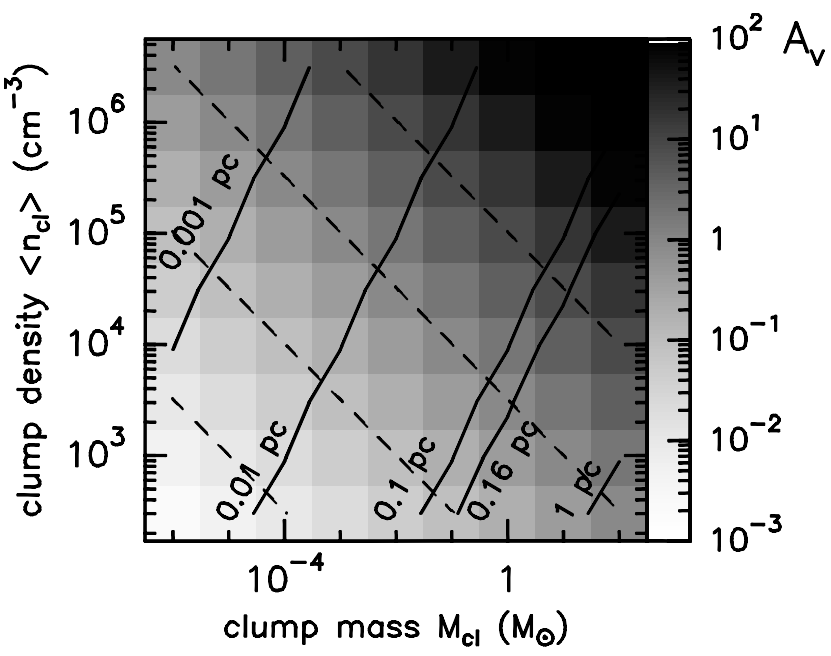

Fig. 9. Grid of $9 \times 9$ PDR models. The average visual extinction for the cross section of each clump is shown as a function of the clump mass and average clump density (grey scales). Dashed contours are drawn for $A_{v}=0.01,0.1,1$, and 10. Solid lines indicate clumps with the same radius. The radius of the SWAS beam at the distance of the B5 cloud is $0.16 \mathrm{pc}(0.5$ times the beam FWHM).

if the velocity dispersion of the clump motion is larger than the (turbulent) velocity governing the line width of the emission in each individual clump. Numerous studies of spectral line radiative transfer in molecular clouds support this macoturbulent view of the cloud structure (see discussion by Falgarone et al. 1998, and references therein). Even for clumps that do overlap in velocity space, it is unlikely that the radiative coupling substantially modifies the intensity of the considered spectral lines. We have seen in Sect. 5.1 that the CO excitation temperature is relatively uniform across the cloud and that the density is large enough to at least thermalize both of the lowest $\mathrm{CO}$ transitions. At these densities, the [CI] fine-structure transition is thermalized, tracing the local temperature structure at the clump surface. In many positions, the density is large enough to thermalize even the $J=3 \rightarrow 2$ transition. Line trapping very likely plays a role for the radiation transfer and the line excitation of a few position close to the cloud edge only. Absorption of the ${ }^{12} \mathrm{CO} J=3 \rightarrow 2$ emission in the lower-density surfaces of foreground clumps might therefore play a role. However, our conclusions are not significantly altered if the $\mathrm{CO} J=3 \rightarrow 2$ data are omitted.

\subsection{PDR model results}

Four model grids are considered for the B5 observations, and the relevant grid parameters are summarized in Table 5. The reference model (grid 1) uses a scaling factor of $\chi=1$ for the FUV field. For grid 2, the FUV field is reduced by $\chi=0.13$. In grids 3 and 4 the spherical clump is surrounded by a halo with a small column density of molecular hydrogen and $\mathrm{CO}$ but no (significant) visual extinction ( $\chi=1$ at the surface of the dense clump). The last two model grids are motivated by the study of Wannier et al. (1999), who detect a small but significant column density of molecular hydrogen in the B5 halo. In addition, these 
Table 5. PDR model grids. For each grid $(9 \times 9$ models $)$ the average clump density $\langle n\rangle_{\mathrm{cl}}$ and clump mass $M_{\mathrm{cl}}$ is varied $\left(2.5 \leq \log \left(\langle n\rangle_{\mathrm{cl}}\right) \leq 6.5\right.$ and $10^{-6} M_{\odot} \leq M_{\mathrm{cl}} \leq 100 M_{\odot}$ ). The range of $\langle n\rangle_{\mathrm{cl}}, M_{\mathrm{cl}}$ that best fits the [CI]/CO line ratios in Table 3 is listed in Cols. 5-10. Note that grids 3 and 4 have additional $\mathrm{H}_{2}$ and $\mathrm{CO}$ pre-shielding column densities (given in the third and fourth columns, respectively), while model grids 1 and 2 have no additional pre-shielding.

\begin{tabular}{|c|c|c|c|c|c|c|c|c|c|}
\hline \multirow{2}{*}{$\begin{array}{c}\text { Grid } \\
\#\end{array}$} & \multirow{2}{*}{$\begin{array}{c}\text { FUV field } \\
\chi\end{array}$} & \multirow{2}{*}{$\begin{array}{l}N_{\mathrm{pre}_{\mathrm{H}}} \\
\left(\mathrm{cm}^{-2}\right)\end{array}$} & \multirow{2}{*}{$\begin{array}{l}N_{\text {pre,CO }} \\
\left(\mathrm{cm}^{-2}\right)\end{array}$} & \multicolumn{2}{|c|}{ B5 core region } & \multicolumn{2}{|c|}{ IRS 4 region } & \multicolumn{2}{|c|}{ B5 edge } \\
\hline & & & & $\log \left(\langle n\rangle_{\mathrm{cl}}\right)$ & $M_{\mathrm{cl}}$ & $\log \left(\langle n\rangle_{\mathrm{cl}}\right)$ & $M_{\mathrm{cl}}$ & $\log \left(\langle n\rangle_{\mathrm{cl}}\right)$ & $M_{\mathrm{cl}}$ \\
\hline 1 & 1 & 0 & 0 & $5.5-6.0$ & $\geq 10^{-4}$ & $5.5-6.0$ & $10^{-5}-1$ & 5.0 & 0.01 \\
\hline 2 & 0.13 & 0 & 0 & $\gtrsim 5.0^{a}$ & ${ }^{a}$ & $5.0-5.5$ & $10^{-5}-0.1$ & 5.0 & $10^{-5}-10^{-4}$ \\
\hline 3 & 1 & $0.63 \times 10^{20}$ & $2.1 \times 10^{13}$ & 4.5 & $\gtrsim 1$ & 4.5 & $\geq 10^{-3}$ & 3.5 & $0.1-1$ \\
\hline 4 & 1 & $1.25 \times 10^{20}$ & $8.5 \times 10^{13}$ & 4.5 & $\gtrsim 10^{2}$ & $4.0-4.5$ & $\gtrsim 1$ & $3.0-3.5$ & $\gtrsim 10$ \\
\hline
\end{tabular}

${ }^{a} \chi^{2}$ surface does not show a well-defined minimum, see Fig. 12.
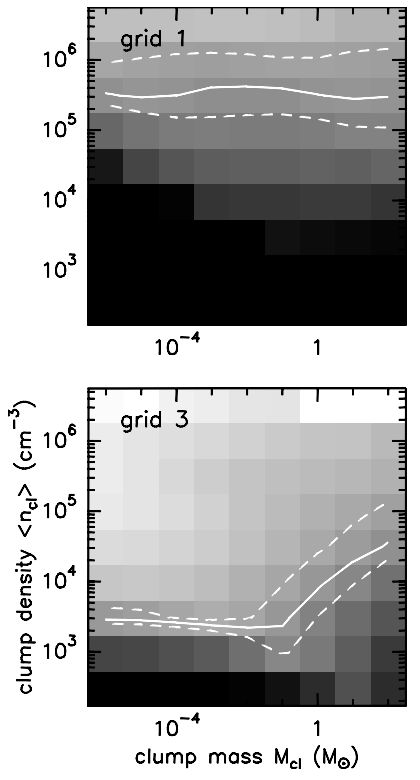

Fig. 10. $[\mathrm{CI}] /{ }^{12} \mathrm{CO} J=2 \rightarrow 1$ line-integrated intensity ratio for PDR model grids $1-4$. Grid $1(\chi=1)$ and grid 2 with a reduced FUV field $(\chi=0.13)$ in the upper panels. The results from the PDR models with pre-shielding and $\chi=1$ are shown in both lower panels (grids 3 and 4). The PDR model results (grey scale) are compared to the observed line ratio for the B5 cloud core region (solid line). The dashed line reflects the $1 \sigma$ accuracy of the observed line ratio, $1.35 \pm 0.35$.

models expand on our previous study where we suggested that a small amount of molecular hydrogen in a diffuse halo or interclump medium might be responsible for the surprisingly small $[\mathrm{CI}] /$ low- $J$ CO line ratios observed in MCLD 123.5+24.9 and other translucent clouds (Bensch et al. 2003).

The $[\mathrm{CI}] / \mathrm{CO}$ line ratio are calculated for the line-integrated intensity of the three lowest ${ }^{12} \mathrm{CO}$ and ${ }^{13} \mathrm{CO}$ rotational transitions for each clump, and these ratios are plotted as a function of $\left\langle n_{\mathrm{cl}}\right\rangle, M_{\mathrm{cl}}$. An example is shown in Fig. 10 with the $[\mathrm{CI}] /{ }^{12} \mathrm{CO} J=2 \rightarrow 1$ ratio. Figure 11 shows additional [CI]/CO line ratios for the simulations in grid 3. Equivalent plots for model grid 1 are presented and discussed by Bensch et al. (2003), and they are not substantially different in the present study. The general trend visible in the plots of Figs. 10 and 11 is the increase of the $[\mathrm{CI}] / \mathrm{CO}$ ratios from the more massive and dense clumps in the upper right corner of the panels to the
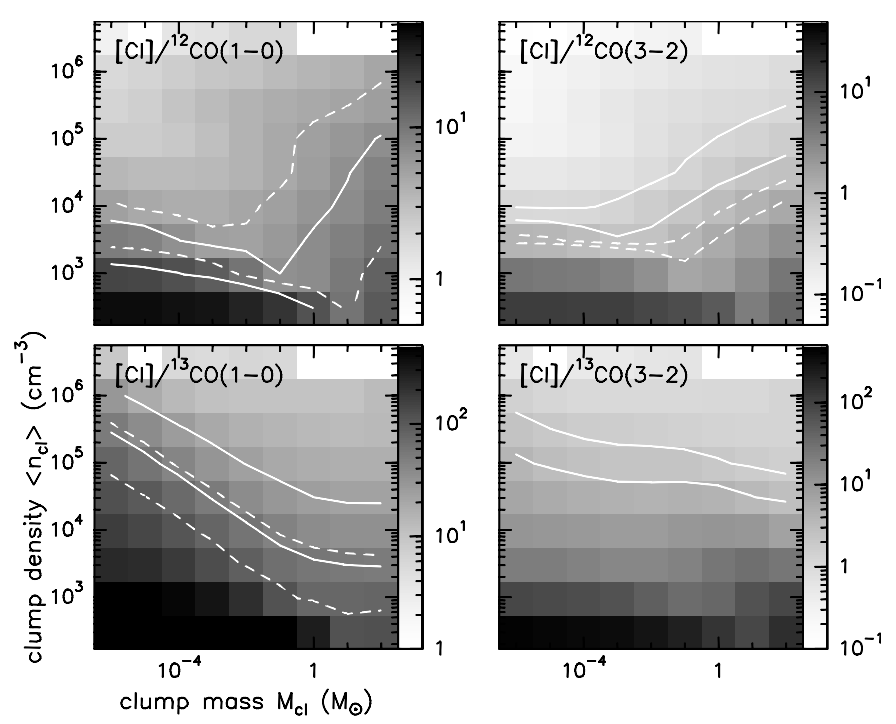

Fig. 11. The PDR model results for grid $3\left(\chi=1, N_{\text {pre, } \mathrm{H}_{2}}=0.63 \times\right.$ $\left.10^{20} \mathrm{~cm}^{-2}\right)$. The four panels on the left compare the observed line ratios (contours) to the results from the PDR simulations. Solid contours give the range of the observed line ratios in the B5 core region, the dashed lines for B5 cloud edge ( $\sigma$ error bars).

less massive and lower density clumps in the lower left. This reflects the lower visual extinction and thus increased photodissociation of $\mathrm{CO}$ in the latter clumps.

For a quantitative comparison of the observations and simulations, the reduced $\chi^{2}$ is calculated for the $[\mathrm{CI}] / \mathrm{CO}$ line ratios observed in three different regions: B5 core, IRS 4, and the cloud edge (upper half of Table 3). Similar to the procedure outlined in Sect. 5.1, the reduced $\chi^{2}$ is calculated as $\chi^{2}=m^{-1} \sum_{i=1}^{m}\left(R_{i, \mathrm{obs}}-R_{i, \mathrm{pdr}}\right)^{2} / \Delta R_{i, \mathrm{obs}}^{2}$, where $R_{i, \mathrm{obs}}, \Delta R_{i, \mathrm{obs}}$ is the observed line ratio and its $1 \sigma$ error, respectively, and $R_{i, \mathrm{pdr}}$ is the line ratio of the PDR model. The summation is over the $[\mathrm{CI}] / \mathrm{CO}$ line ratios calculated for the three lowest rotational transitions of ${ }^{12} \mathrm{CO}$ and ${ }^{13} \mathrm{CO}$. The resulting $\chi^{2}$ is drawn as a function of average clump mass and clump density. This is shown in Fig. 12 for the model grids 1 to 4 and the observations toward the B5 core region and cloud edge. For each model grid and set of observed line ratios, a global minimum of the order of unity can be identified for the $\chi^{2}\left(\langle n\rangle_{\mathrm{cl}}, M_{\mathrm{cl}}\right)$ surface. The exception is grid 2 and the observations in the B5 core region 

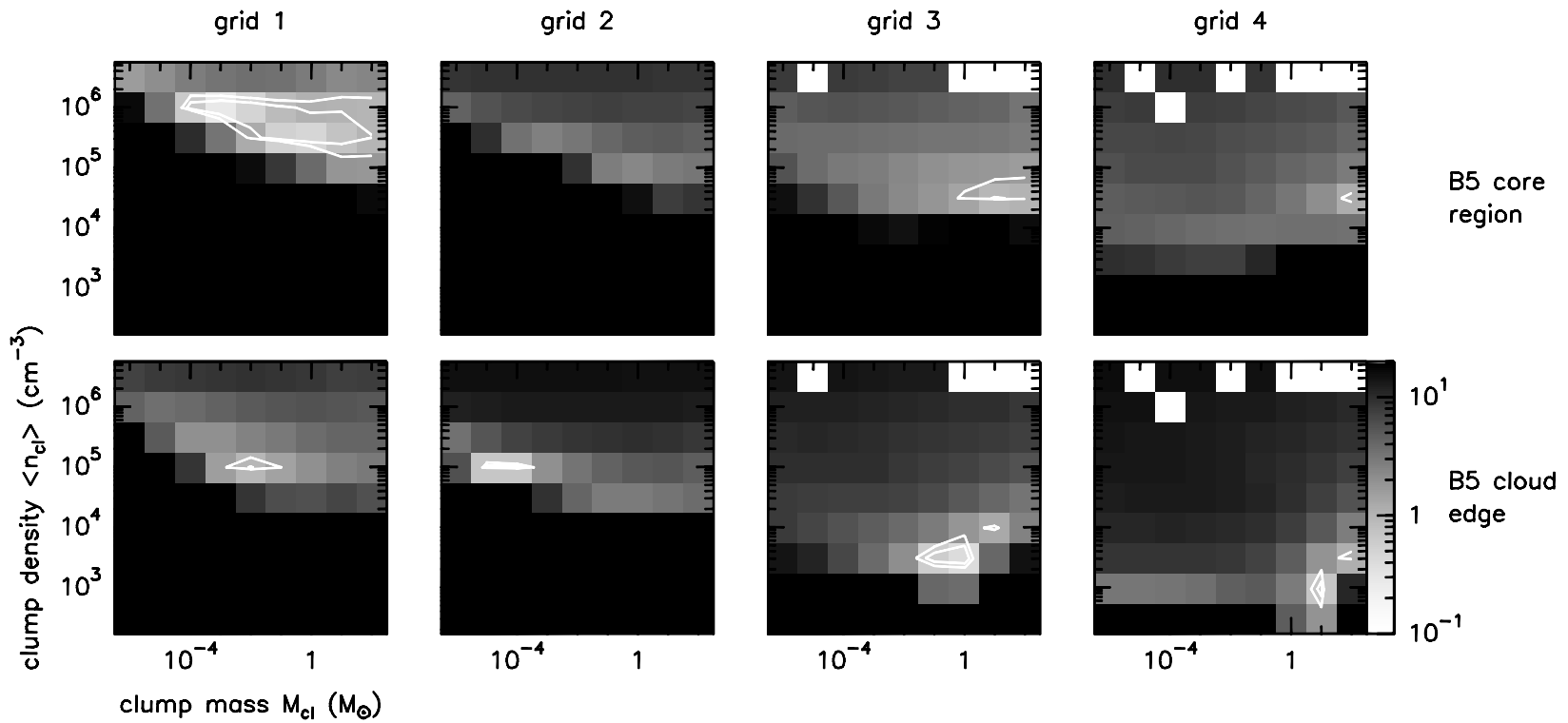

Fig. 12. The PDR model results for the $[\mathrm{CI}] / \mathrm{CO}$ line ratios observed in B5. The reduced $\chi^{2}$ for PDR grids $1-4$ and the line ratios observed in the B5 core region (upper row of panels) and the cloud edge (bottom row). Contours are drawn for $\chi^{2}=1$ and 1.5 (see text for details).

where $\chi_{\text {min. }}^{2}=2.4$, indicating that none of the PDR models in grid 2 can reproduce the observations toward the B5 core.

For the B5 core region, the best-matching models in grid 1 have $\log \left\langle n_{\mathrm{cl}}\right\rangle=5.0-6.0$ and cover a wide range in clump mass. Much lower densities are implied by the models with $\mathrm{H}_{2}$ pre-shielding, $\log \left\langle n_{\mathrm{cl}}\right\rangle \sim 4.5$ (for grid 3) and $\log \left\langle n_{\mathrm{cl}}\right\rangle \sim 4.0$ (grid 4), with $M_{\mathrm{cl}} \gtrsim 1 M_{\odot}$ in both cases. Similar results are obtained for the line ratios observed in the IRS 4 region. Slightly smaller densities and clump masses are derived for the cloud edge (Table 5).

\subsection{Discussion of the results}

The PDR simulations demonstrate that a small column density of molecular hydrogen $\left(\$ 10^{20} \mathrm{~cm}^{-2}\right)$ in a halo surrounding a clump of denser gas substantially changes the $[\mathrm{CI}] /{ }^{12} \mathrm{CO}$ and $[\mathrm{CI}] /{ }^{13} \mathrm{CO}$ line ratios of that clump. The $\mathrm{CO}$ is dissociated via line-absorption to a pre-dissociation state. Several of these lines overlap in frequency with transitions of molecular hydrogen. Therefore, molecular hydrogen efficiently shields $\mathrm{CO}$, in addition to the $\mathrm{CO}$ self-shielding. This effect is considerably augmented if $\mathrm{H}_{2}$ (and $\mathrm{CO}$ ) is present in a lower-density halo surrounding the clump. The impact is greater for clumps with a low-density $\left(\left\langle n_{\mathrm{cl}}\right\rangle \lesssim 10^{3} \mathrm{~cm}^{-3}\right)$ where the $[\mathrm{CI}] /{ }^{12} \mathrm{CO}$ line ratios are reduced by up to three orders of magnitude. This results from the significantly larger $\mathrm{CO}$ abundance in these clumps, whereas the $\mathrm{C}^{0}$ abundance (and thus the $[\mathrm{CI}]$ emission) is significantly reduced. For clumps with a higher density, the preshielding shifts the $\mathrm{C}^{0} / \mathrm{CO}$ transition closer to the clump surface and reduces the total $\mathrm{C}^{0}$ column density, again resulting in a weaker $[\mathrm{CI}]$ and stronger $\mathrm{CO}$ emission.

The low- $J{ }^{12} \mathrm{CO}$ and ${ }^{13} \mathrm{CO}$ line ratios, in contrast, change less dramatically for models with pre-shielding. (The exceptions are clumps with a low visual extinction where the overall $\mathrm{CO}$ abundance is increased by the pre-shielding.) This indicates that the gas temperature as a function of radius is relatively constant where $\mathrm{CO}$ is the most abundant carbon species and that the gas temperature experiences only a small variation resulting from the different abundance of the primary gas coolants $\mathrm{C}^{+}, \mathrm{C}^{0}$, and $\mathrm{CO}$. Consequently, the low- $J$ CO line ratios show only a relatively small variation as a function of the clump parameter, and the observed line ratios are reproduced by clumps with a wide range of mass and density. A similar result has been noted by Störzer et al. (2000) for clumps in stronger FUV fields.

For each grid, a range of models can be identified that reproduce the observed $[\mathrm{CI}] / \mathrm{CO}$ line ratios. However, only the "best-fit" model in grid 3 is consistent with additional, independent constraints for the density, visual extinction, and the spatial extent of the emission. The PDR model in grid 3 indicates typical clump densities of $\log \langle n\rangle_{\mathrm{cl}} \sim 4.5$ for the core and IRS 4 region. The small difference in the line ratios for both regions indicate that the clump mass in the core region is $M_{\mathrm{cl}} \gtrsim 10 M_{\odot}$, and thus somewhat larger than in the IRS 4 region $\left(M_{\mathrm{cl}} \approx 0.1-10 M_{\odot}\right)$. For the cloud edge, the observed line ratios suggest clumps with a lower density $\log \langle n\rangle_{\mathrm{cl}} \sim 3.5$ and a lower clump mass $\left(M_{\mathrm{cl}} \approx 0.1-1 M_{\odot}\right)$. The rather high density of $\log \langle n\rangle_{\mathrm{cl}} \gtrsim 5.5$ indicated by the models in grids 1 and 2 might apply for the B5 core, but it is rather unlikely for the bulk of the gas throughout the cloud (Bensch et al. 2003). At least for the cloud edge, these densities are in conflict with the results from the escape-probability study in Sect. 5.1, as well as the literature data summarized in Sect. 2. In addition, the peak temperatures of the models in grid $2(\chi=0.13)$ are smaller by factor of $2-8$ than what is observed toward the core and IRS 4 region, even for those models which match the observed line ratios (Table 2). This suggests that the low observed 
Table 6. Peak temperature (in Kelvins): observations and PDR model predictions for the B5 core region.

\begin{tabular}{|c|c|c|c|c|c|}
\hline transition & $\begin{array}{c}\text { grid 1 } \\
\log \left\langle n_{\mathrm{cl}}\right\rangle=6.0 \\
M_{\mathrm{cl}}=0.001 M_{\odot}\end{array}$ & $\begin{array}{c}\text { grid } 2 \\
\log \left\langle n_{\mathrm{cl}}\right\rangle=5.0 \\
M_{\mathrm{cl}}=1.0 M_{\odot}\end{array}$ & $\begin{array}{c}\text { grid } 3 \\
\log \left\langle n_{\mathrm{cl}}\right\rangle=4.5 \\
M_{\mathrm{cl}}=10 M_{\odot}\end{array}$ & $\begin{array}{c}\text { grid } 4 \\
\log \left\langle n_{\mathrm{cl}}\right\rangle=4.5 \\
M_{\mathrm{cl}}=100 M_{\odot}\end{array}$ & $\begin{array}{c}\text { observed: } \\
\text { B5 core } \\
\text { region }\end{array}$ \\
\hline$[\mathrm{CII}]^{2} \mathrm{P}_{3 / 2} \rightarrow{ }^{2} \mathrm{P}_{1 / 2}$ & $3.5 \times 10^{-3}$ & $2.3 \times 10^{-5}$ & $5.4 \times 10^{-2}$ & $4.1 \times 10^{-2}$ & \\
\hline$[\mathrm{CI}]{ }^{3} \mathrm{P}_{1} \rightarrow{ }^{3} \mathrm{P}_{0}$ & 1.56 & 0.55 & 1.75 & 1.78 & $1.3 \pm 0.3$ \\
\hline$[\mathrm{CI}]{ }^{3} \mathrm{P}_{2} \rightarrow{ }^{3} \mathrm{P}_{1}$ & 0.23 & 0.01 & 0.25 & 0.21 & \\
\hline $\mathrm{CO} J=1 \rightarrow 0$ & 8.22 & 3.50 & 10.85 & 11.23 & $9.3 \pm 3.5$ \\
\hline $\mathrm{CO} J=2 \rightarrow 1$ & 7.47 & 2.02 & 6.56 & 7.30 & $8.1 \pm 1.0$ \\
\hline $\mathrm{CO} J=3 \rightarrow 2$ & 5.57 & 0.85 & 3.38 & 4.17 & $6.5 \pm 0.8$ \\
\hline $\mathrm{CO} J=4 \rightarrow 3$ & 3.28 & 0.37 & 1.46 & 1.82 & \\
\hline $\mathrm{CO} J=5 \rightarrow 4$ & 1.11 & 0.04 & 0.32 & 0.50 & \\
\hline $\mathrm{CO} J=6 \rightarrow 5$ & 0.10 & $<0.01$ & 0.014 & 0.05 & \\
\hline${ }^{13} \mathrm{CO} J=1 \rightarrow 0$ & 3.44 & 3.23 & 5.19 & 6.01 & $5.0 \pm 1.8$ \\
\hline${ }^{13} \mathrm{CO} J=2 \rightarrow 1$ & 2.99 & 1.98 & 3.28 & 3.85 & $3.5 \pm 0.5$ \\
\hline${ }^{13} \mathrm{CO} J=3 \rightarrow 2$ & 1.53 & 0.70 & 1.30 & 1.82 & $2.2 \pm 0.4$ \\
\hline${ }^{13} \mathrm{CO} J=4 \rightarrow 3$ & 0.35 & 0.05 & 0.20 & 0.46 & \\
\hline${ }^{13} \mathrm{CO} J=5 \rightarrow 4$ & 0.04 & $<0.01$ & 0.01 & 0.03 & $<0.3$ \\
\hline
\end{tabular}

$[\mathrm{CI}] / \mathrm{CO}$ ratio does not result from a general attenuation of the FUV field.

For grid 4 with the larger pre-shielding column densities, the clumps that match the observed line ratios have an average visual extinction exceeding that of the observed $A_{\mathrm{v}}$, and radii that are larger than the projected diameter of the beam. Thus, they are in conflict with the observed small-scale structure and visual extinction toward B5.

The pre-shielding column density in grid 3 is a factor of 2-3 smaller than implied by the UV absorption measurements by Wannier et al. (1999). Even though the absorption measurements only provide the information along a pencil beam, and the derived $\mathrm{H}_{2}$ column densities vary by a factor of $\sim 2$ for the four background stars, they suggest column densities of typically a few times $10^{20} \mathrm{~cm}^{-2}$ and thus pre-shielding column densities of $N_{\mathrm{H}_{2} \text {,pre }} \sim(1-2) \times 10^{20} \mathrm{~cm}^{-2}$ on either side of the cloud. The smaller pre-shielding might indicate that the $\mathrm{H}_{2}$ in the halo is not uniformly distributed. Also, a fraction of the $\mathrm{H}_{2}$ detected in the halo by means of UV-absorption measurements resides in a second diffuse cloud that is not associated with B5 (see Kaczmarczyk 2000 and Fig. 3).

Observations of additional transitions can be used to test the PDR models. Table 6 shows the peak temperature predicted for the B5 core region by the best-fitting model in each grid. The beam filling factor is expected to be close to unity for the core region, and the observed peak intensity can be directly compared to these predictions. The higher $\mathrm{CO}$ transitions with their higher critical densities are very useful for discriminating between models with and without pre-shielding. Since the models without pre-shielding require clumps of substantially larger density, they predict a much stronger CO $J=4 \rightarrow 3$ and $J=5 \rightarrow 4$ emission. The ${ }^{12} \mathrm{CO} J=4 \rightarrow 3$ can be detected relatively easily with ground-based radio telescopes. The $J=5 \rightarrow 4$ transition is even a more sensitive discriminant among the four sets of models; however, it is not accessible to ground-based telescopes. The $[\mathrm{CI}]{ }^{3} \mathrm{P}_{2} \rightarrow{ }^{3} \mathrm{P}_{1}$ transition is sensitive to the temperature at the clump surface and thus the strength of the external FUV field.

\section{Summary}

Observations of radio-line emission are presented for B5, a relatively isolated dark cloud in the Perseus molecular cloud complex. The spectral line data encompass mapping observations for the three lowest rotational transitions of ${ }^{12} \mathrm{CO}$ and ${ }^{13} \mathrm{CO}$, complemented by deeply integrated spectra $\left(t_{\mathrm{on}} \leq 20 \mathrm{~h}\right)$ of the $[\mathrm{CI}]{ }^{3} \mathrm{P}_{1} \rightarrow{ }^{3} \mathrm{P}_{0}$ fine-structure transition of neutral carbon. The CO maps are elongated in declination and extend from the cloud core to the northern cloud edge and into the surrounding halo. The $[\mathrm{CI}]$ observations are made with SWAS for 12 pointings along a $1 \mathrm{D}$ cut with the same orientation as the $\mathrm{CO}$ maps. The visual extinction along the cut varies from $A_{\mathrm{v}} \gtrsim 7$ toward the cloud core to $A_{\mathrm{v}} \lesssim 1$ in the halo.

The emission from the three lowest ${ }^{12} \mathrm{CO}$ transition and the ${ }^{13} \mathrm{CO} J=1 \rightarrow 0$ transition are detected across the entire area mapped, including the low- $A_{\mathrm{v}}$ halo. The ${ }^{13} \mathrm{CO} J=2 \rightarrow 1$, $J=3 \rightarrow 2$, and the [CI] transition, in contrast, are detected only for the region with strong ${ }^{12} \mathrm{CO}$ emission. The $\mathrm{CO}$ excitation in the halo is dominated by the absorption of line photons emitted by the B5 molecular cloud. This might help to explain the spatially extended ${ }^{12} \mathrm{CO}$ emission detected in a halo where the density is much smaller than the critical density of the rotational lines. The $[\mathrm{CI}]$ emission, in contrast, is not detected in the cloud halo, even though neutral carbon is more abundant than CO. This results from the line excitation of neutral carbon, which is governed by collisions even at the low halo densities of a factor of ten below the critical density of the [CI] transition. An upper limit to the neutral carbon column density of $N_{\mathrm{C}^{0}}<6 \times 10^{15} \mathrm{~cm}^{-2}$ is derived for the halo, somewhat larger than the column density derived by Wannier et al. (1999) from UV absorption-line measurements for four lines-of-sight with an angular distance of $\gtrsim 0.5^{\circ}$ to the cloud. This suggests that the halo is not spherically symmetric, because we expect 
a significantly larger column density in the halo for the SWAS lines-of-sight in this case. Future observations will provide the sensitivity needed to detect and map the [CI] sub-mm emission in molecular cloud halos (ground-based observations at very high and dry sites, and air- and space-based observations).

The UV absorption measurements by Savage et al. (1977) and Wannier et al. (1999) reveal the presence of molecular hydrogen in the halo surrounding B5 with typical $\mathrm{H}_{2}$ column densities of a few times $10^{20} \mathrm{~cm}^{-2}$. The PDR simulations presented here demonstrate that $\mathrm{H}_{2}$ column densities of this magnitude in a surrounding halo significantly modify the $\mathrm{CO}$ and $[\mathrm{CI}]$ line emission of molecular clouds. This pre-shielding increases the $\mathrm{CO}$ line intensity and decreases the $[\mathrm{CI}]$ intensity, and thus is particularly prominent in the $[\mathrm{CI}] / \mathrm{CO}$ line ratios. For the low rotational transitions of ${ }^{12} \mathrm{CO}$ and ${ }^{13} \mathrm{CO}$, the line ratios can change by a factor of two (for clumps with $n \sim 10^{4} \mathrm{~cm}^{-3}$ ), and up to three orders of magnitude (for $n<10^{3} \mathrm{~cm}^{-3}$ ). The possible influence of this effect has to be considered if the [CI]/CO line ratios are interpreted in the framework of a PDR.

For the B5 cloud, PDR models with $\chi=1$ and a preshielding column density of $N_{\mathrm{pre}, \mathrm{H}_{2}} \sim 0.6 \times 10^{20} \mathrm{~cm}^{-2}$ are found to match the observed $[\mathrm{CI}] /{ }^{12} \mathrm{CO}$ and $[\mathrm{CI}] /{ }^{13} \mathrm{CO}$ line ratio, as well as other observational constraints for B5 (linear extent of the emitting regions, visual extinction). These models imply a clump mass of $M_{\mathrm{cl}} \gtrsim 1 M_{\odot}$ and a density of $\langle n\rangle_{\mathrm{cl}} \sim 3 \times 10^{4} \mathrm{~cm}^{-3}$ for the region close to the B5 core. Clumps of similar density but smaller mass dominate the emission north of the core, near the infrared source IRS 4 . The clumps at the northern cloud edge have a lower density and mass, $n \sim 3 \times 10^{3} \mathrm{~cm}^{-3}$ and $0.1 M_{\odot} \lesssim M_{\mathrm{cl}} \lesssim 1 M_{\odot}$. Models with a globally attenuated FUV field, in contrast, fail to reproduce the observed peak temperatures. In addition, they suggest densities that are in conflict with independently derived constraints on the gas density.

The PDR model predicts that the gas cooling of the clumps in $\mathrm{B} 5$ cloud is dominated by the $\mathrm{CO}$ rotational transitions, whereas the [CII] fine-structure transition dominates the cooling of the molecular gas in clumps close to the cloud edge. The fine-structure transitions of neutral carbon, in contrast, play only a minor role. Additional observations of the $\mathrm{CO}$ $J=4 \rightarrow 3$ and $J=5 \rightarrow 4$ rotational transitions and of the [CI] ${ }^{3} \mathrm{P}_{2} \rightarrow{ }^{3} \mathrm{P}_{1}$ and [CII] fine-structure transitions can be used to test this picture. However, stratospheric and/or spacebased observatories with up-to-date instrumentation are required to detect these spectral lines. The exception is the ${ }^{12} \mathrm{CO}$ $J=4 \rightarrow 3$ transition, which can be detected with ground-based telescopes.

Acknowledgements. I want to thank Paul F. Goldsmith and C. Kramer for critically reading the manuscript, and Robert Simon for conducting a significant part of the KOSMA observations presented in the paper. Also, I want to thank the anonymous referee for the constructive remarks on the manuscript. This research is supported by the Deutsche Forschungsgemeinschaft DFG (research stipend Be 2412/1-1 and Be 2412/1-2) and by the National Aeronautics and Space Administration NASA through an AAS small research grant. The FCRAO is supported by NSF grant AST 02-28993. The KOSMA telescope on Gornergrat is jointly operated by the Universities of Cologne and Bonn, and supported by a special fund from the
Land Nordrhein-Westfalen. The observatory is administered by the Internationale Stiftung Hochalpine Forschungsstationen Jungfraujoch und Gornergrat. This research made use of NASA's Astrophysics Data System Abstract Service and the SIMBAD database operated at the CDS, Strasbourg, France.

\section{References}

Andersson, B.-G., Roger, R. S., \& Wannier, P. G. 1992, A\&A, 260, 355

Andersson, B.-G., \& Wannier, P. G. 1993, ApJ, 402, 585

Beichman, C. A., Jennings, R. E., Emerson, J. P., et al. 1984, ApJ, 278, L45

Bensch, F., Stutzki, J., \& Heithausen, A. 2001a, A\&A, 365, 285

Bensch, F., Panis, J.-F., Stutzki, J., Heithausen, A., \& Falgarone, E. 2001b, A\&A, 365, 275

Bensch, F., Leuenhagen, U., Stutzki, J., \& Schieder, R. 2003, ApJ, 591, 1013

Benson, P. J., \& Myers, P. C. 1989, ApJS, 71, 89

Boissé, P. 1990, A\&A, 228, 483

C̆ernis, K. 1993, Baltic Astronomy, vol. 2, 214

The COMPLETE Survey of Star-Forming Regions, cfa-www.harvard.edu/COMPLETE

Dickey, J. M., \& Lockman, F. J. 1990, ARA\&A, 28, 215

Dickman, R. L. 1978, ApJS, 37, 407

Downes, D. 1989, Introductory courses in Galaxies (Springer)

Draine, B. T. 1978, ApJS, 36, 595

Falgarone, E., Panis, J.-F., Heithausen, A., et al. 1998, A\&A, 331, 669

Federman, S. R., Glassgold, A. E., Jenkins, E. B., \& Shaya, E. J. 1980, ApJ, 242, 545

Flower, D. R., \& Launay, J. M. 1985, MNRAS, 214, 271

Frerking, M. A., Keene, J., Blake, G. A., \& Phillips, T. G. 1989, ApJ, 344,311

Fuller, G. A., Myers, P. C., Welch, W. J., et al. 1991, ApJ, 376, 135

Gierens, K. M., Stutzki, J., \& Winnewisser, G. 1992, A\&A, 259, 271

Goldsmith, P. F., Langer, W. D., \& Wilson, R. W. 1986, ApJ, 303, L11

Goodman, A. A. 2004, in Star Formation in the Interstellar Medium, ASP Conf. Ser., San Francisco, in press

Green, S., \& Thaddeus, P. 1976, ApJ, 205, 766

Herbig, G. H., \& Jones, B. F. 1983, AJ, 88, 1040

Jenkins, E. B., Jura, M., \& Loewenstein, M. 1983, ApJ, 270, 88

Jenkins, E. B., \& Tripp, T. M. 2001, ApJS, 137, 297

Kaczmarczyk, G. 2000, MNRAS, 316, 875

Kamegai, K., Ikeda, M., Maezawa, H., et al. 2003, ApJ, 589, 378

Keene, J., Blake, G. A., \& Phillips, T. G. 1987, ApJ, 313, 396

Kulkarni, S. R., \& Heiles, C. 1987, in Interstellar Processes, ed. D. Hollenbach, \& H. A. Thronson Jr. (Dordrecht: Reidel), 87

Langer, W. D., Wilson, R. W., Goldsmith, P. F., \& Beichman, C. A. 1989, ApJ, 337, 355

Launay, J. M., \& Roueff, E. 1977, A\&A, 56, 289

Le Teuff, Y. H., Millar, T. J., \& Markwick, A. J. 2000, A\&AS, 146, 157

Maezawa, H., Ikeda, M., Ito, T., et al. 1999, ApJ, 524, L129

McKee, C. F., Hollenbach, D., \& Wolfire, M. G. 2004, in The Dense Interstellar Medium in Galaxies, ed. Pfalzner et al., Proc. of the 4th Cologne-Bonn-Zermatt-Symposium, (Berlin: Springer Verlag), 395

Melnick, G. J., Stauffer, J. R., Ashby, M. L. N., et al. 2000, ApJ, 539, L77 
Moriarty-Schieven, G. H., Andersson, B.-G., \& Wannier, P. G. 1997, ApJ, 475, 642

Rachford, B. L., Snow, T. P., Tumlinson, J., et al. 2002, ApJ, 577, 221

Savage, B. D., Bohlin, R. C., Drake, J. F., \& Budich, W. 1977, ApJ, 216, 291

Schilke, P., Keene, J., Le Bourlot, J., Pineau des Forêts, G., \& Roueff, E., A\&A, 294, L17

Shull, J. M., \& Beckwith, S. 1982, ARA\&A, 20, 163

Spitzer Jr., L., \& Jenkins, E. B. 1975, ARA\&A, 13, 133

Stark, R., Wesselius, P. R., van Dishoeck, E. F., \& Laureijs, R. J. 1996, A\&A, 311, 282

Störzer, H., Stutzki, J., \& Sternberg, A. 1996, A\&A, 310, 592

Störzer, H., Zielinsky, M., Stutzki, J., \& Sternberg, A. 2000, A\&A, 358,682

Stutzki, J., \& Winnewisser, G., A\&A, 144, 13

Sun, K., Kramer, C., Bensch, F., Stutzki, J., \& Miller, M. 2005, A\&A, submitted

Tatematsu, K., Jaffe, D. T., Plume, R., \& Evans II, N. J. 1999, ApJ, 526,295
Tielens, A. G. G. M. 2001, Preparatory work for the Herschel Space Observatory, collected and edited by Xander Tielens; available on http://www.sron.rug.nl/hifiscience/

Ungerechts, H., \& Thaddeus, P. 1987, ApJS, 63, 645

van Dishoeck, E. F., \& Black, J. H. 1988, ApJ, 334, 771

Wannier, P. G., Penprase, B. E., \& Andersson, B.-G. 1997, ApJ, 487, L165

Wannier, P. G., Andersson, B.-G., Penprase, B. E., \& Federman, S. R. 1999, ApJ, 510, 291

Warin, S., Benayoun, J. J., \& Viala, Y. P. 1996, A\&A, 308, 535

Wolfire, M., McKee, C. F., Hollenbach, D., \& Tielens, A. G. G. M. 2003, ApJ, 587, 278

Young, J. S., Goldsmith, P. F., Langer, W. D., Wilson, R. W., \& Carlson, E. R. 1982, ApJ, 261, 513

Yu, K. C., Billawala, Y., \& Bally, J. 1999, AJ, 118, 2940

Zhou, S., Wu, Y., Evans II, N. J., Fuller, G. A., \& Myers, P. C. 1989, ApJ, 346, 168

Zielinsky, M., Stutzki, J., \& Störzer, H. 2000, A\&A, 358, 723 